\title{
High-Order Solution Methods for Grey Discrete Ordinates Thermal Radiative Transfer
}

\author{
Peter G. Maginot ${ }^{\mathrm{a}, *}$, Jean C. Ragusa ${ }^{\mathrm{b}}$, Jim E. Morel ${ }^{\mathrm{b}}$ \\ ${ }^{a}$ Lawrence Livermore National Laboratory, Livermore, CA 94551, USA \\ ${ }^{b}$ Department of Nuclear Engineering, Texas A $6 M$ University, College Station, TX 77843, \\ $U S A$
}

\begin{abstract}
This work presents a solution methodology for solving the grey radiative transfer equations that is both spatially and temporally more accurate than the canonical radiative transfer solution technique of linear discontinuous finite element discretization in space with implicit Euler integration in time. We solve the grey radiative transfer equations by fully converging the nonlinear temperature dependence of the material specific heat, material opacities, and Planck function. The grey radiative transfer equations are discretized in space using arbitraryorder self-lumping discontinuous finite elements and integrated in time with arbitrary-order diagonally implicit Runge-Kutta time integration techniques. Iterative convergence of the radiation equation is accelerated using a modified interior penalty diffusion operator to precondition the full discrete ordinates transport operator.
\end{abstract}

Keywords: thermal radiation transport, discontinuous finite element method, Runge-Kutta, discrete ordinates

\section{Introduction}

The goal of this work is to accurately solve the time-dependent thermal radiative transfer (TRT) equations. The TRT equations are a nonlinear system of

\footnotetext{
* Corresponding author

Email addresses: maginot1@1lnl.gov (Peter G. Maginot), jean.ragusa@tamu.edu (Jean C. Ragusa), morel@tamu.edu (Jim E. Morel)
}

Preprint submitted to Journal of Computational Physics

September 9, 2016

(C) 2016. This manuscript version is made available under the Elsevier user license http://www.elsevier.com/open-access/userlicense/1.0/ 
equations that describe the conservation and transfer of energy between a photon (radiation) field and a stationary material. Solution of the TRT equations is an important component in several areas of physics including, but not limited to, astrophysics, inertial confinement fusion, and high energy density laboratory physics experiments.

It has previously been shown for linear neutron transport spatially discretized with discontinuous Galerkin finite elements (DFEM) that an increase of the trial space polynomial degree results in increased accuracy per unknown as compared to lower degree polynomial trial space DFEM solutions [1]. Additionally, as computing hardware advances, the number of floating point operations (FLOPs) available per memory operation continues to increase [2]. Computer architecture is not yet to the point where the higher number of FLOPs per unknown associated with higher order methods is completely masked by memory latency and relative narrowing of memory bandwidth $[3,4,5]$. However, we are quickly approaching an era in computing where increased accuracy through increased FLOPs per unknown will no longer result in a corresponding increase in time to solution; the main computing performance bottleneck will likely be memory latency or memory bandwidth.

In radiative transfer, material absorption opacities are strong functions of temperature; the common assumption being that opacities, $\sigma$, vary proportionally to $\frac{1}{T^{3}}[6,7,8,9]$. Since temperature varies within each spatial zone, accurate TRT spatial discretization schemes must explicitly account for this within-zone opacity variation. As shown in [10] for neutron transport, the common zonewise constant cross section approximation limits DFEM order of convergence to at most second order in space, regardless of trial space degree.

We use an overall solution strategy similar to that of Morel et al. [11] to solve the grey thermal radiative transfer equations: discrete ordinates to treat the angular dependence of the radiation equations, quasi-Newton iteration on material temperature, and approximation of the Planck function spatial dependence as a polynomial contained within the DFEM trial space. However, our work varies from that of [11] in several ways. First, in this work we use arbitrary-order 
self-lumping DFEM [10] rather than limiting our derivation to lumped linear DFEM. Likewise, we derive our equations using arbitrary-order, arbitrary stage count diagonally implicit Runge-Kutta (SDIRK) time integration [12], rather than considering only first order, single stage, implicit Euler time integration. Additionally, we fully converge the nonlinear temperature dependence of the grey TRT at every time integration stage, rather than performing a single quasiNewton iteration. Each quasi-Newton iteration fully accounts for the nonlinear temperature dependence of the Planck function. Material property nonlinear dependencies are resolved by updating all temperature dependent material properties with every new temperature iterate. New temperature iterates come from the linearization of the Planck function temperature dependence. When the Planck linearization is fully converged, the nonlinear dependencies of the material properties are also converged. Finally, we do not assume that opacity and heat capacity are zone-wise constant; we explicitly account for the within zone variation of material properties via our self-lumping DFEM [10].

The remainder of this paper is divided as follows. In Section 2 we derive the discrete ordinate, grey thermal radiative transfer equations spatially discretized with arbitrary order DFEM and integrated in time with an arbitrary stage count SDIRK method. Additionally in Section 2, we apply the modified interior penalty diffusion synthetic operator $[13,14]$ to accelerate the iterative convergence of our linearized arbitrary order DFEM radiation equation. An overview of our solution algorithm is given in Section 3. In Section 4 we present a series computational problems and results that: verify our implementation, demonstrate the asymptotic orders of convergence of our new methodology, show that the proposed methods yield tangible benefits for physically meaning full simulations. In Section 4 we also briefly discuss the computational performance of our algorithm. Finally, we discuss our results and offer concluding remarks in Section 5. 


\section{Discretization of the Thermal Radiative Transfer Equations}

We begin with the slab geometry, grey, thermal radiative transfer equations:

$$
\begin{gathered}
\frac{1}{c} \frac{\partial I}{\partial t}+\mu \frac{\partial I}{\partial x}+\sigma_{t} I=\frac{\sigma_{s}}{4 \pi} \phi+\sigma_{a} B+Q_{I} \\
C_{v} \frac{\partial T}{\partial t}=\sigma_{a}(\phi-4 \pi B)+Q_{T},
\end{gathered}
$$

where $x$ is position in the slab; $t$ is time; $c$ is the speed of light; $I$ is the frequency integrated intensity, $I=I(x, \mu, t) ; \mu$ is the directional cosine of the intensity relative to the $x$ axis; $T$ is the material temperature, $T=T(x, t) ; \phi$ is the frequency and angle integrated intensity, $\phi=\phi(x, t) ; \sigma_{t}, \sigma_{s}$, and $\sigma_{a}$ are respectively the total, scattering, and absorption opacities, with each opacity being a function of space and material temperature; $B$ is the Planck function, $B(T) ; C_{v}$ is the material heat capacity, $C_{v}=C_{v}(x, T) ; Q_{I}$ is a driving radiation source, $Q_{I}=Q_{I}(x, \mu, t) ;$ and $Q_{T}$ is a driving temperature source, $Q_{T}=Q_{T}(x, t)$. Driving sources are considered for generality and the use of the method of manufactured solutions. In Eq. (1a), we have assumed only isotropic scattering, and define the angle integrated intensity as:

$$
\phi(x)=2 \pi \int_{-1}^{1} I(x, \mu) d \mu .
$$

The frequency integrated Planck function, with Planck radiation constant, $a$, is:

$$
B(T)=\frac{a c T^{4}}{4 \pi} .
$$

Introducing the discrete ordinates approximation,

$$
\phi \approx 2 \pi \sum_{d=1}^{N_{d i r}} \omega_{d} I_{d},
$$

where $\mu_{d}, \omega_{d}$ are direction and weight pairs of a given angular quadrature set with $\sum_{d}^{N_{d i r}} \omega_{d}=2, I_{d}=I\left(\mu_{d}\right)$, and $Q_{I_{d}}=Q_{I}\left(\mu_{d}\right)$, Eq. (1a) becomes:

$$
\frac{1}{c} \frac{\partial I_{d}}{\partial t}+\mu_{d} \frac{\partial I_{d}}{\partial x}+\sigma_{t} I_{d}=\frac{\sigma_{s}}{4 \pi} \phi+\sigma_{a} B+Q_{I_{d}}
$$


To advance the grey thermal radiative transfer equations in time, we use arbitrary order (with arbitrary stage count) S-stable diagonally implicit RungeKutta (SDIRK) time integration schemes [12], alternatively referred to as single diagonally implicit Runge-Kutta time integration schemes, depending on the reference cited [15]. Alexander defines that an S-stable diagonally implicit Runge-Kutta approximation, $y^{n}(t)$, of time dependent function, $g(t)$, will obey

$$
\frac{\left|g\left(t^{n+1}\right)-y^{n+1}\right|}{\left|g\left(t^{n}\right)-y^{n}\right|}<1
$$

where $t$ is time, $y^{n}$ is the numerical estimate of $g(t)$ at time $t^{n}, t^{n+1}>t^{n}$, and $g(t)$ is a bounded function with finite derivatives [12].

Prior to temporally discretizing the grey TRT, we detail how SDIRK methods solve initial value problems of the form

$$
\begin{aligned}
g\left(t^{n}\right) & =g^{n} \\
\frac{\partial g}{\partial t} & =f(t, g) .
\end{aligned}
$$

An $N_{s}$ stage SDIRK scheme is described by coefficients $\alpha_{i j}, \beta_{j}$, and $\gamma_{i}$ that are typically given in a Butcher tableaux of the form

\begin{tabular}{c|c|cccc} 
Stage & $\gamma_{i}$ & $\alpha_{i j}$ & & & \\
\hline 1 & $\gamma_{1}$ & $\alpha_{11}$ & 0 & $\ldots$ & 0 \\
2 & $\gamma_{2}$ & $\alpha_{21}$ & $\alpha_{22}$ & 0 & $\vdots$ \\
$i$ & $\gamma_{i}$ & $\alpha_{i 1}$ & $\alpha_{i 2}$ & $\ddots$ & 0 \\
$N_{s}$ & $\gamma_{N_{s}}$ & $\alpha_{N_{s}} 1$ & $\alpha_{N_{s}} 2$ & $\ldots$ & $\alpha_{N_{s} N_{s}}$ \\
\hline & & $\beta_{1}$ & $\beta_{2}$ & $\ldots$ & $\beta_{N_{s}}$
\end{tabular}

Given $y^{n}$, SDIRK methods compute the next time step solution as:

$$
y^{n+1}=y^{n}+\Delta t \sum_{i=1}^{N_{s}} \beta_{i} k^{i}
$$

where $\Delta t=t^{n+1}-t^{n}$, and $k^{i}$ is defined as:

$$
k^{i}=f\left(t^{n}+\gamma_{i} \Delta t, y^{n}+\Delta t \sum_{j=1}^{i} \alpha_{i j} k^{j}\right) \text {. }
$$


Equation (10) can be manipulated to yield:

$$
y^{i}=y^{n}+\Delta t \sum_{j=1}^{i} \alpha_{i j} f\left(t^{j}, y^{j}\right)
$$

where $y^{i}$ is the intermediate estimate of $g$ at the time of stage $i$ and $t^{j}=$ $t^{n}+\Delta t \gamma_{j}$. We use the $i$ notation throughout the remainder of the paper to indicate quantities evaluated at time stage $i$. We consider three specific SDIRK time differencing schemes:

1. implicit Euler: one stage, first order accurate;

2. SDIRK 2-2: two stages, second order accurate;

3. SDIRK 3-3: three stages, third order accurate.

The Butcher tableaux for each method is given in Appendix A.

We apply SDIRK time integration to the grey thermal radiative transfer equations beginning with a form equivalent to Eq. (11),

$$
\begin{gathered}
I_{d}^{i}=I_{d}^{n}+\Delta t \sum_{j=1}^{i-1} \alpha_{i j} k_{I_{d}}^{j} \\
+\alpha_{i i} \Delta t c\left[\frac{\sigma_{s}}{4 \pi} \phi^{i}+\sigma_{a} B\left(T^{i}\right)-\sigma_{t} I_{d}^{i}-\mu_{d} \frac{\partial I_{d}^{i}}{\partial x}+Q_{I_{d}}^{i}\right] \\
T^{i}=T^{n}+\Delta t \sum_{j=1}^{i-1} \alpha_{i j} k_{T}^{j}+\frac{\alpha_{i i} \Delta t}{C_{v}}\left\{\sigma_{a}\left[\phi^{i}-4 \pi B\left(T^{i}\right)\right]+Q_{T}^{i}\right\} .
\end{gathered}
$$

In Eqs. (12), we have used the definitions of the time rates of change of intensity, $k_{I_{d}}^{i}$, and temperature, $k_{T}^{i}$, evaluated at time $t^{i}$ :

$$
\begin{gathered}
k_{I_{d}}^{i}=\left.\frac{\partial I_{d}}{\partial t}\right|_{t=t^{i}}=c\left[\frac{\sigma_{s}}{4 \pi} \phi^{i}+\sigma_{a} B\left(T^{i}\right)-\sigma_{t} I_{d}^{i}-\mu_{d} \frac{\partial I_{d}^{i}}{\partial x}+Q_{I_{d}}^{i}\right] \\
k_{T}^{i}=\left.\frac{\partial T}{\partial t}\right|_{t=t^{i}}=\frac{1}{C_{v}}\left\{\sigma_{a}\left[\phi^{i}-4 \pi B\left(T^{i}\right)\right]+Q_{T}^{i}\right\} .
\end{gathered}
$$

Next, we linearize the Planck function in temperature as follows:

$$
B\left(T^{i}\right) \approx B^{*}+D^{*}\left[T^{i}-T^{*}\right],
$$


where

$$
\begin{aligned}
B^{*} & =B\left(T^{*}\right) \\
D^{*} & =D\left(T^{*}\right) \\
D\left(T^{*}\right) & =\left.\frac{d B}{d T^{\prime}}\right|_{T^{\prime}=T^{*}},
\end{aligned}
$$

and $T^{*}$ being an arbitrary temperature iterate. This transforms Eqs. (12) to:

$$
\begin{aligned}
& I_{d}^{i}=I_{d}^{n}+\Delta t \sum_{j=1}^{i-1} \alpha_{i j} k_{I_{d}}^{j} \\
& +\alpha_{i i} \Delta t c\left\{\frac{\sigma_{s}}{4 \pi} \phi^{i}+\sigma_{a}\left[B^{*}+D^{*}\left(T^{i}-T^{*}\right)\right]-\sigma_{t} I_{d}^{i}-\mu_{d} \frac{\partial I_{d}^{i}}{\partial x}+Q_{I_{d}}^{i}\right\} \\
& T^{i}=T^{n}+\Delta t \sum_{j=1}^{i-1} \alpha_{i j} k_{T}^{j} \\
& \quad+\frac{\alpha_{i i} \Delta t}{C_{v}}\left(\sigma_{a}\left\{\phi^{i}-4 \pi\left[B^{*}+D^{*}\left(T^{i}-T^{*}\right)\right]\right\}+Q_{T}^{i}\right)
\end{aligned}
$$

where we implicitly note:

$$
\begin{gathered}
k_{I_{d}}^{i} \approx c\left\{\frac{\sigma_{s}}{4 \pi} \phi^{i}+\sigma_{a}\left[B^{*}+D^{*}\left(T^{i}-T^{*}\right)\right]-\sigma_{t} I_{d}^{i}-\mu_{d} \frac{\partial I_{d}^{i}}{\partial x}+Q_{I_{d}}^{i}\right\} \\
k_{T}^{i} \approx \frac{1}{C_{v}}\left(\sigma_{a}\left\{\phi^{i}-4 \pi\left[B^{*}+D^{*}\left(T^{i}-T^{*}\right)\right]\right\}+Q_{T}^{i}\right) .
\end{gathered}
$$

In Eqs. (17), the " $\approx$ " is used to denote the linearization of the Planck function in temperature. Finally, following a procedure similar to that outlined by Morel et al. [11], we:

1. spatially discretize Eqs. (16),

2. algebraically manipulate the spatially discretized variant of Eq. (16b) to obtain an expression for the temperature at time $t^{i}$,

3. insert this definition of $T^{i}$ into the spatially discretized form of Eq. (16a), and

4. manipulate this final equation into a form reminiscent of a spatially discretized linear neutron transport equation with fission. 
The complete algebraic manipulation is given in Appendix B, but we provide highlights of the derivation here.

We define a $P$ degree polynomial DFEM trial space, using $N_{P}=P+1$ basis functions, $b_{\ell}(s)$, in every spatial zone, $z, z \in\left[1, N_{z}\right]$. In this work, a spatial zone is synonymous with a spatial cell. Our interpolatory polynomial basis functions are defined on a reference element, such that physical zone $z$, defined on $x \in\left[x_{z-1 / 2}, x_{z+1 / 2}\right]$, is mapped to $s \in[-1,1]$. We map from the reference element to the physical domain with a linear transformation,

$$
\begin{aligned}
x & =x_{z}+\frac{\Delta x_{z}}{2} s \\
x_{z} & =\frac{x_{z-1 / 2}+x_{z+1 / 2}}{2} \\
\Delta x_{z} & =x_{z+1 / 2}-x_{z-1 / 2} .
\end{aligned}
$$

Each Lagrangian basis function interpolates at a unique $s_{\ell}$, such that $-1 \leq$ $s_{1}<\cdots<s_{\ell}<\cdots<s_{N_{P}} \leq 1$, and is defined on the reference element as:

$$
b_{\ell}(s)=\prod_{\substack{m=1 \\ m \neq \ell}}^{N_{P}} \frac{s-s_{m}}{s_{\ell}-s_{m}}
$$

Following the standard Galerkin procedure we multiply Eqs. (16) by every basis function and integrate over the domain, yielding $N_{P} \times N_{d i r} \times N_{z}$ radiation equations and $N_{P} \times N_{z}$ temperature equations. To close the system of equations, we assume solution representations equal to a linear combination of our chosen basis functions. For intensity,

$$
I_{d}^{i}(x) \approx \widetilde{I}_{d}^{i}(x)=\sum_{z=1}^{N_{z}}\left[\sum_{\ell=1}^{N_{P}} I_{d, z, \ell}^{i} b_{\ell}(s)\right] .
$$

Angle integrated intensity and temperature are expanded in an analogous fashion to Eq. (20). Likewise, there are spatially discretized variants of the time rates of change of intensity and temperature. As an example, the intensity time rate of change expansion is

$$
k_{I_{d}}^{i}(x) \approx \widetilde{k}_{I_{d}}^{i}(x)=\sum_{z=1}^{N_{z}}\left[\sum_{\ell=1}^{N_{P}} k_{I_{d}, z, \ell}^{i} b_{\ell}(s)\right] .
$$


One of the fundamental assumptions of our method is the approximation of the spatial dependence of the Planck function, $B(T(x))$, as,

$$
B^{i}\left(T^{i}(x)\right) \approx \widetilde{B}_{i}\left(\widetilde{T}^{i}(x)\right)=\sum_{z=1}^{N_{z}}\left[\sum_{\ell=1}^{N_{P}} B\left(T_{z, \ell}^{i}\right) b_{\ell}(s)\right] .
$$

Because each basis function has support only in spatial mesh zone $z$, we may consider each zone independently (with appropriate interface conditions). We now give the spatially discretized variants of the intensity and temperature time rates of change,

$$
\begin{aligned}
& \frac{1}{c} \mathbf{M} \vec{k}_{I}^{i}=\frac{1}{4 \pi} \mathbf{R}_{\sigma_{s}} \vec{\phi}^{i}+\mathbf{R}_{\sigma_{a}}\left[\vec{B}^{*}+\mathbf{D}^{*}\right.\left.\left(\vec{T}^{i}-\vec{T}^{*}\right)\right] \\
&-\mathbf{R}_{\sigma_{t}} \vec{I}^{i}+\mu_{d} I_{i n} \vec{u}-\mu_{d} \mathbf{G} \vec{I}^{i}+\vec{Q}_{I}^{i} \\
& \mathbf{R}_{C_{v}} \vec{k}_{T}^{i}=\mathbf{R}_{\sigma_{a}}\left\{\vec{\phi}^{i}-4 \pi\left[\vec{B}^{*}+\mathbf{D}^{*}\left(\vec{T}^{i}-\vec{T}^{*}\right)\right]\right\}+\vec{Q}_{T}^{i} .
\end{aligned}
$$

In Eqs. (23), we define the vectors of unknowns in zone $z$, at time $t^{i}$, for direction $d$ (as appropriate), as:

$$
\begin{aligned}
\vec{I}^{i} & =\left[I_{d, z, 1}^{i}, \ldots, I_{d, z, N_{P}}^{i}\right]^{T} \\
\vec{\phi}^{i} & =\left[\phi_{z, 1}^{i}, \ldots, \phi_{z, N_{P}}^{i}\right]^{T} \\
\vec{T}^{i} & =\left[T_{z, 1}^{i}, \ldots, T_{z, N_{P}}^{i}\right]^{T} \\
\vec{k}_{I}^{i} & =\left[k_{I_{d}, z, 1}^{i}, \ldots, k_{I_{d}, z, N_{P}}^{i}\right]^{T} \\
\vec{k}_{T}^{i} & =\left[k_{T, z, 1}^{i}, \ldots, k_{T, z, N_{P}}^{i}\right]^{T} .
\end{aligned}
$$

The definition of the known temperature iterate, $\vec{T}^{*}$, is analogous to Eq. (24c). $\vec{B}^{*}$ is defined as

$$
\vec{B}^{*}=\left[B\left(T_{z, 1}^{*}\right), \ldots, B\left(T_{z, N_{P}}^{*}\right)\right]^{T} .
$$

The assumption that the spatial variation of the Planck emission term can be expressed in the DFEM trial space allows us to write the Planck linearization in temperature as

$$
\vec{B}_{i}=\vec{B}^{*}+\mathbf{D}^{*}\left(\vec{T}^{i}-\vec{T}^{*}\right)
$$


where $\mathbf{D}^{*}$ is a $N_{P} \times N_{P}$ diagonal matrix, with elements, $\mathbf{D}_{\ell m}^{*}$ defined as:

$$
\mathbf{D}_{\ell m}^{*}=\left\{\begin{array}{ll}
\left.\frac{d B}{d T}\right|_{T=T^{*}, z, \ell} & \ell=m \\
0 & \text { otherwise }
\end{array} .\right.
$$

In Eqs. (23), $\mathbf{M}$ is the $N_{P} \times N_{P}$ mass matrix,

$$
\mathbf{M}_{\ell m}=\frac{\Delta x_{z}}{2} \int_{-1}^{1} b_{\ell}(s) b_{m}(s) d s,
$$

and $\mathbf{R}_{v}$ are reaction matrices defined as

$$
\mathbf{R}_{v, \ell m}=\frac{\Delta x_{z}}{2} \int_{-1}^{1} v\left[s, \widetilde{T}_{z}^{*}(s)\right] b_{\ell}(s) b_{m}(s) d s,
$$

where $v$ is a spatially- and/or temperature-dependent material property $\left(\sigma_{a}, \sigma_{s}\right.$, $\sigma_{t}$, or $C_{v}$ ), evaluated using known temperature iterate $T^{*}$. In Eq. (23a), we have integrated the gradient of the angular intensity by parts and used upwinding on the zone edges. On zone edges, $\partial_{z}$, the angular intensity, $I\left(\partial_{z}\right)$ is defined as:

$$
I\left(\partial_{z}\right)=\left\{\begin{array}{lr}
\widetilde{I}_{d, z}^{i}\left(\partial_{z}\right) & \mu_{d} \cdot \vec{n}_{\partial_{z}}>0 \\
I_{i n} & \text { otherwise }
\end{array} .\right.
$$

In Eq. (30), $\vec{n}_{\partial_{z}}$ is the outward directed edge normal and $I_{i n}$ is a known angular intensity outflow from a previous zone or a known boundary condition. Thus, the gradient matrix $\mathbf{G}$ and inflow vector $\vec{u}$ are defined as:

$$
\begin{aligned}
\mathbf{G}_{\ell m} & =s g\left(\mu_{d}\right) b_{\ell}\left[s g\left(\mu_{d}\right)\right] b_{m}\left[s g\left(\mu_{d}\right)\right]-\int_{-1}^{1} \frac{\partial b_{\ell}}{\partial s} b_{m}(s) d s \\
\vec{u}_{\ell} & =s g\left(\mu_{d}\right) b_{\ell}\left[-s g\left(\mu_{d}\right)\right]
\end{aligned}
$$

where

$$
s g(\mu)=\left\{\begin{array}{ll}
+1 & \mu>0 \\
-1 & \mu<0
\end{array} .\right.
$$

Finally, the $N_{P}$ length vectors of driving source moments, $\vec{Q}_{I}^{i}$ and $\vec{Q}_{T}^{i}$, are defined as

$$
\begin{aligned}
\vec{Q}_{I, \ell}^{i} & =\frac{\Delta x_{z}}{2} \int_{-1}^{1} b_{\ell}(s) Q_{I_{d}}^{i}(s) d s \\
\vec{Q}_{T, \ell}^{i} & =\frac{\Delta x_{z}}{2} \int_{-1}^{1} b_{\ell}(s) Q_{T}^{i}(s) d s .
\end{aligned}
$$


We chose to not divide by $C_{v}(s)$ in Eq. (23b) before discretizing for several reasons:

1. to account for a more natural quantity, $C_{v}(s)$, rather than $\frac{1}{C_{v}(s)}$,

2. to re-use $\mathbf{R}_{C_{v}}$ when computing an energy balance, and

3. to yield a more elegant spatial discretization than the alternative.

Using the spatially discretized versions of the intensity and temperature time derivatives, the spatially discretized, SDIRK expressions for the time $t^{i}$ intensity and temperature in zone $z$ are:

$$
\begin{aligned}
\vec{I}^{i}=\vec{I}^{n}+\Delta t \sum_{j=1}^{i-1} \alpha_{i j} \vec{k}_{I}^{j}+\frac{1}{4 \pi}\left[\alpha_{i i} \Delta t c \mathbf{M}^{-1}\right] \mathbf{R}_{\sigma_{s}} \vec{\phi}^{i} \\
+\alpha_{i i} \Delta t c \mathbf{M}^{-1} \mathbf{R}_{\sigma_{a}}\left[\vec{B}^{*}+\mathbf{D}^{*}\left(\vec{T}^{i}-\vec{T}^{*}\right)\right] \\
\quad+\alpha_{i i} \Delta t c \mathbf{M}^{-1}\left[\mu_{d} I_{i n} \vec{u}-\mathbf{R}_{\sigma_{t}} \vec{I}^{i}-\mu_{d} \mathbf{G} \vec{I}^{i}+\vec{Q}_{I}^{i}\right] \\
\vec{T}^{i}=\vec{T}^{n}+\Delta t \sum_{j=1}^{i-1} \alpha_{i j} \vec{k}_{T}^{j} \\
\quad+\alpha_{i i} \Delta t \mathbf{R}_{C_{v}}^{-1}\left(\mathbf{R}_{\sigma_{a}}\left\{\vec{\phi}^{i}-4 \pi\left[\vec{B}^{*}+\mathbf{D}^{*}\left(\vec{T}^{i}-\vec{T}^{*}\right)\right]\right\}+\vec{Q}_{T}^{i}\right) .
\end{aligned}
$$

Algebraic manipulation of Eqs. (35), detailed in Appendix B, yields a temperature update equation,

$$
\begin{aligned}
\vec{T}^{i}=\vec{T}^{*}+\mathbf{C}^{i}\left[\vec{T}^{n}-\vec{T}^{*}+\right. & \left.\Delta t \sum_{j=1}^{i-1} \alpha_{i j} k_{T}^{j}\right] \\
& +\Delta t \alpha_{i i} \mathbf{C}^{i} \mathbf{R}_{C_{v}}^{-1}\left[\mathbf{R}_{\sigma_{a}}\left(\vec{\phi}^{i}-4 \pi \vec{B}^{*}\right)+\vec{Q}_{T}^{i}\right] .
\end{aligned}
$$

In Eq. (36), we have used a coefficient matrix, $\mathbf{C}^{i}$, defined as

$$
\mathbf{C}^{i}=\left[\mathbf{I}+4 \pi \Delta t \alpha_{i i} \mathbf{R}_{C_{v}}^{-1} \mathbf{R}_{\sigma_{a}} \mathbf{D}^{*}\right]^{-1}
$$

with I the $N_{P} \times N_{P}$ identity matrix. Inserting Eq. (36) into Eq. (35a) and manipulating yields, for a given temperature iterate $T^{*}$, a linear transport equation equivalent to a linear neutron transport equation with scattering, fission, 
and fixed sources,

$$
\mu_{d} \mathbf{G} \vec{I}^{\imath}+\mathbf{R}_{\sigma_{\tau}}^{i} \vec{I}^{i}=\frac{1}{4 \pi} \mathbf{R}_{\sigma_{s}} \vec{\phi}^{i}+\frac{1}{4 \pi} \overline{\bar{\nu}}^{i} \mathbf{R}_{\sigma_{a}} \vec{\phi}^{i}+\vec{\xi}^{\imath}+\mu_{d} \vec{u} I_{i n},
$$

as detailed in Appendix B. In Eq. (38), $N_{P} \times N_{P}$ matrices $\overline{\bar{\nu}}^{i}$ and $\mathbf{R}_{\sigma_{\tau}}^{i}$ are defined as:

$$
\begin{gathered}
\overline{\bar{\nu}}^{i}=4 \pi \Delta t \alpha_{i i} \mathbf{R}_{\sigma_{a}} \mathbf{D}^{*} \mathbf{C}^{i} \mathbf{R}_{C_{v}}^{-1} \\
\mathbf{R}_{\sigma_{\tau}}^{i}=\mathbf{R}_{\sigma_{t}}+\frac{1}{c \Delta t \alpha_{i i}} \mathbf{M}
\end{gathered}
$$

and $N_{P} \times 1$ vector $\vec{\xi}^{\imath}$ is defined as

$$
\begin{aligned}
\vec{\xi}^{\imath}=\frac{1}{c \Delta t \alpha_{i i}} \mathbf{M} \overrightarrow{I^{n}} & +\frac{1}{c \alpha_{i i}} \mathbf{M} \sum_{j=1}^{i-1} \alpha_{i j} \vec{k}_{I}^{j}+\mathbf{R}_{\sigma_{a}} \vec{B}^{*}+\vec{Q}_{I}^{i} \\
+ & \mathbf{R}_{\sigma_{a}} \mathbf{D}^{*} \mathbf{C}^{i} \\
& {\left[\vec{T}^{n}-\vec{T}^{*}+\Delta t \sum_{j=1}^{i-1} \alpha_{i j} k_{T}^{j}\right] } \\
& +\Delta t \alpha_{i i} \mathbf{R}_{\sigma_{a}} \mathbf{D}^{*} \mathbf{C}^{i} \mathbf{R}_{C_{v}}^{-1}\left[\vec{Q}_{T}^{i}-4 \pi \mathbf{R}_{\sigma_{a}} \vec{B}^{*}\right] .
\end{aligned}
$$

We emphasize the following points here,

1. arbitrary order/stage count SDIRK time integration applied to the thermal radiative transfer equations is very similar to using implicit Euler time integration and

2. the equations we have derived hold for any discontinuous finite element scheme that can be written as a linear system of equations, regardless of DFEM trial space.

\subsection{Self-Lumping Discontinuous Finite Elements Methods}

In this paper, we consider quadrature based self-lumping discontinuous finite element methods. Self-lumping DFEM (SL DFEM) automatically create diagonal mass matrices by using numerical quadrature restricted to the interpolation points of the DFEM basis functions $[16,17]$. The choice of DFEM interpolation point greatly affects the behavior of SL DFEM. We restrict ourselves in this work to SL DFEM schemes that use Gauss-Legendre (Gauss) or Gauss-LobattoLegendre (Lobatto) quadrature as the DFEM interpolation points. Unlike other 
choices of DFEM interpolation point, SL DFEM schemes using Gauss or Lobatto interpolation points have been shown to converge the $L_{2}$ norm of the error in angular flux and interaction rates $\propto P+1$ and to be more robust $[10,18]$.

We consider four SL DFEM schemes in total:

1. SL Lobatto: Lobatto quadrature interpolation points, zone-wise constant material properties,

2. SL Gauss: Gauss quadrature interpolation points, zone-wise constant material properties,

3. SLXS Lobatto: Lobatto quadrature interpolation points, evaluation of material properties at quadrature points, and

4. SLXS Gauss: Lobatto quadrature interpolation points, evaluation of material properties at quadrature points.

All four DFEM schemes evaluate $\mathbf{G}$ and $\mathbf{M}$ using a quadrature rule

$$
\mathbf{G}_{\ell m}=b_{\ell}\left(s g\left(\mu_{d}\right)\right) b_{m}\left(s g\left(\mu_{d}\right)\right)-\left.\frac{\Delta x_{z}}{2} \sum_{q=1}^{N_{P}} w_{q} \frac{d b_{\ell}}{d s}\right|_{s=s_{q}} b_{m}\left(s_{q}\right),
$$

and

$$
\mathbf{M}_{\ell m}=\frac{\Delta x_{z}}{2} \sum_{q=1}^{N_{P}} w_{q} b_{\ell}\left(s_{q}\right) b_{m}\left(s_{q}\right)
$$

where $\left\{s_{q}, w_{q}\right\}$ are the $N_{P}$ quadrature point and weight pairs that define a numerical quadrature. Due to the interpolatory nature of the basis functions,

$$
b_{\ell}\left(s_{m}\right)=\left\{\begin{array}{rr}
1 & \ell=m \\
0 & \text { otherwise }
\end{array},\right.
$$

by allowing the DFEM interpolation points to be the same as the numerical quadrature points, Eq. (42) is equivalent to

$$
\mathbf{M}_{\ell m}=\left\{\begin{array}{ll}
w_{\ell} \frac{\Delta x_{z}}{2} & \ell=m \\
0 & \text { otherwise }
\end{array} .\right.
$$

All four SL DFEM schemes will exactly evaluate $\mathbf{G}$, whereas the SL DFEM with Lobatto interpolation points will approximately integrate $\mathbf{M}$ and the SL DFEM with Gauss interpolation points will exactly integrate $\mathbf{M}$ [18]. 
The SL Lobatto and SL Gauss schemes approximate $\mathbf{R}_{v}$ as:

$$
\mathbf{R}_{v} \approx \bar{v} \mathbf{M}
$$

where $\bar{v}$ is a zone-wise volumetric average of material property of $z$. SLXS Lobatto and SLXS Gauss evaluate $\mathbf{R}_{v}$ as:

$$
\mathbf{R}_{v, \ell m}=\left\{\begin{array}{lr}
v\left(s_{\ell}, T_{z, \ell}^{*}\right) w_{\ell} \frac{\Delta x_{z}}{2} & \ell=m \\
0 & \text { otherwise }
\end{array} .\right.
$$

It can be shown that using the linear SL Lobatto scheme with implicit Euler time integration and only performing one Newton iteration per time step yields the [traditionally] lumped linear discontinuous equations of Morel et. al [11], after the equations in [11] are integrated over all photon energy groups. This is expected given

1. the results in [18] that demonstrated the equivalence of traditional lumping and self-lumping Lobatto DFEM schemes for discontinuous linear finite elements and

2. that we used the same process as outlined in [11].

\subsection{Modified Interior Penalty Synthetic Acceleration}

The standard method of solving Eq. (38) for the intensity for a given temperature is Richardson or source iteration [19],

$$
\begin{gathered}
\mu_{d} \mathbf{G} \vec{I}^{\vec{i}(\ell+1)}+\mathbf{R}_{\sigma_{\tau}}^{i} \vec{I}^{i,(\ell+1)}=\frac{1}{4 \pi} \mathbf{R}_{\sigma_{s}} \vec{\phi}^{i,(\ell)}+\frac{1}{4 \pi} \overline{\bar{\nu}}^{i} \mathbf{R}_{\sigma_{a}} \vec{\phi}^{i,(\ell)}+\vec{\xi}^{\vec{\imath}}+\mu_{d} \vec{u} I_{i n}^{(\ell+1)} \\
\phi^{i,(\ell+1)}=\sum_{d} w_{d} I_{d}^{i,(\ell+1)}
\end{gathered}
$$

where $(\ell)$ denotes the $\ell$-th iterate of a given quantity within a source iteration. Unfortunately, due to the absorption and re-emission of photons within a time step, as in many thermal radiative transfer problems of interest, the iterative process of Eq. (47) converges arbitrarily slowly [11].

As in [11] we use the diffusion synthetic acceleration (DSA) [20] technique developed to accelerate the iterative convergence of linear neutron transport 
problems to accelerate the iterative convergence of the linearized thermal radiative transfer intensity equations. For the grey TRT case, photon scattering and photon absorption/re-emission can be accelerated simultaneously. In multifrequency TRT, diffusion preconditioning is typically used at two levels: DSA is used to accelerate the within group scattering and linearized multi-frequency grey acceleration is used to accelerate the absorption/re-emission of photons within a time step. DSA for linear neutron transport accelerates problems of the form,

$$
\mu_{d} \frac{\partial \psi_{d}}{\partial x}+\Sigma_{t} \psi_{d}=\frac{1}{4 \pi} \Sigma_{s} \Phi+Q_{\psi_{d}}
$$

using the related diffusion problem,

$$
-\nabla \cdot D \nabla \Phi+\Sigma_{a} \Phi=Q_{\Phi}
$$

In Eq. (48) and Eq. (49), $\psi$ is the angular flux, analogous to the photon intensity, $\Phi$ is the scalar flux, analogous to the angle integrated intensity, $\Sigma_{t}, \Sigma_{s}$, and $\Sigma_{a}$, are the total, scattering, and absorption macroscopic cross sections, $D$ is the diffusion coefficient,

$$
D=\frac{1}{3 \Sigma_{t}}
$$

$Q_{\psi_{d}}$ is an angle dependent source, and $Q_{\Phi}$ is an angle integrated source:

$$
Q_{\Phi}=2 \pi \sum_{d} w_{d} Q_{\psi_{d}}
$$

To be effective, a DSA diffusion operator must be consistent with the radiation transport spatial discretization [21]. For maximum computational benefit, the DSA diffusion operator must also be efficiently invertible [22]. Though several diffusion operator spatial discretizations meet our requirements of consistency and efficient inversion in slab geometry $[22,23,24]$, we wish to use a diffusion operator that, when applied to multi-dimensional geometries, remains iteratively effective on computational meshes with highly skewed zones and is computationally efficient to invert. The modified interior penalty (MIP) diffusion operator discretization $[13,14]$ meets both criteria. 
As detailed in [13], to use the MIP diffusion operator to accelerate the iterative convergence of our linearized radiation equations requires us to be able to evaluate a local stiffness matrix, $\mathbf{H}$,

$$
\mathbf{H}_{\ell m}=\frac{\Delta x_{z}}{2} \int_{-1}^{1} \widehat{D}(s) b_{\ell}(s) b_{m}(s) d s
$$

weighted by effective diffusion coefficient, $\widehat{D}(s)$,

$$
\widehat{D}(s)=\frac{1}{3 \widehat{\Sigma}_{t}(s)}
$$

where $\widehat{\Sigma}_{t}$,

$$
\widehat{\Sigma_{t}}(s)=\frac{1}{\alpha_{i i} c \Delta t}+\sigma_{t}(s) .
$$

Equation (38) defines an effective total macroscopic reaction matrix, $\mathbf{R}_{\sigma_{\tau}}^{i}$, and an effective macroscopic scattering reaction matrix, $\mathrm{x}$

$$
\mathbf{R}_{\widehat{\Sigma}_{s}}=\overline{\bar{\nu}}^{i} \mathbf{R}_{\sigma_{a}}+\mathbf{R}_{\sigma_{s}}
$$

Thus, we naturally define the local absorption matrix for the diffusion operator as

$$
\mathbf{R}_{\widehat{\Sigma}_{a}}=\mathbf{R}_{\sigma_{\tau}}^{i}-\overline{\bar{\nu}}^{i} \mathbf{R}_{\sigma_{a}}-\mathbf{R}_{\sigma_{s}} .
$$

For linear neutron transport, the spectral radius for the iterative convergence of Eqs. (47) approaches the material scattering ratio, $c_{\text {scat }}[21]$,

$$
c_{\text {scat }}=\frac{\Sigma_{s}}{\Sigma_{t}} .
$$

Then for Eq. (38), we introduce the effective scattering matrix, $\mathbf{S}^{i}$,

$$
\mathbf{S}^{i}=\left[\mathbf{R}_{\sigma_{\tau}}^{i}\right]^{-1}\left[\overline{\bar{\nu}}^{i} \mathbf{R}_{\sigma_{a}}+\mathbf{R}_{\sigma_{s}}\right]
$$

Using the evaluations of $\mathbf{R}_{\sigma_{a}}, \mathbf{R}_{\sigma_{s}}, \mathbf{R}_{C_{v}}, \mathbf{R}_{\sigma_{\tau}}^{i}$, and $\overline{\bar{\nu}}^{i}$, given in Appendix B 
for the case of SL Lobatto with implicit Euler time integration, $\mathbf{S}^{i}$ becomes:

$$
\begin{aligned}
& \mathbf{S}^{i}=\left(\frac{c \Delta t}{1+c \Delta t \bar{\sigma}_{t}}\right) \operatorname{diag}\left[\bar{\sigma}_{s}+\bar{\sigma}_{a}\left(\frac{\left.4 \pi \Delta t \bar{\sigma}_{a} \frac{\partial B}{\partial T}\right|_{T=T_{z, 1}^{*}}}{\bar{C}_{v}+\left.4 \pi \Delta t \bar{\sigma}_{a} \frac{\partial B}{\partial T}\right|_{T=T_{z, 1}^{*}}}\right)\right. \\
& \left.\bar{\sigma}_{s}+\bar{\sigma}_{a}\left(\frac{\left.4 \pi \Delta t \bar{\sigma}_{a} \frac{\partial B}{\partial T}\right|_{T=T_{z, 2}^{*}}}{\bar{C}_{v}+\left.4 \pi \Delta t \bar{\sigma}_{a} \frac{\partial B}{\partial T}\right|_{T=T_{z, 2}^{*}}}\right)\right] .
\end{aligned}
$$

For nearly all problems of interest (i.e $1 \ll c \Delta t \bar{\sigma}_{t}$ and $C_{v} \ll 4 \pi \Delta t \bar{\sigma}_{a} \frac{\partial B}{\partial T}$ ) it is clear that the eigenvalues of $\mathbf{S}^{i}$ are very close to unity, thus the need for an iterative acceleration technique.

\section{Algorithm Overview}

As shown in Fig. 1, our solution method for the grey TRT equations can be thought of as a nested iterative process with four loops, with time being the outermost loop, the SDIRK stages per time step being the second level, the nonlinear temperature iteration being the second lowest level loop, and the iterative process required to solve for an updated intensity being the innermost loop. In the remainder of this section, we elaborate on features and requirements of our solution algorithm.

\subsection{Memory Requirements and Nonlinear Thermal Iteration}

First, we discuss the memory requirements of our algorithm. To avoid storing extra copies of the angle-dependent intensity (the largest memory requirement), on the innermost loop we save only the angle integrated intensity, not the full intensity solution at each iterate, $(\ell)$. Since the temperature update equation, Eq. (36), only requires the angle integrated intensity, saving only the angle integrated intensity is permissible. However, this requires one additional transport sweep when calculating the stage $i$ partial derivative with respect to time of the DFEM approximation of intensity, $\widetilde{k}_{I_{d}}^{i}$, at the end of each time stage iteration, 


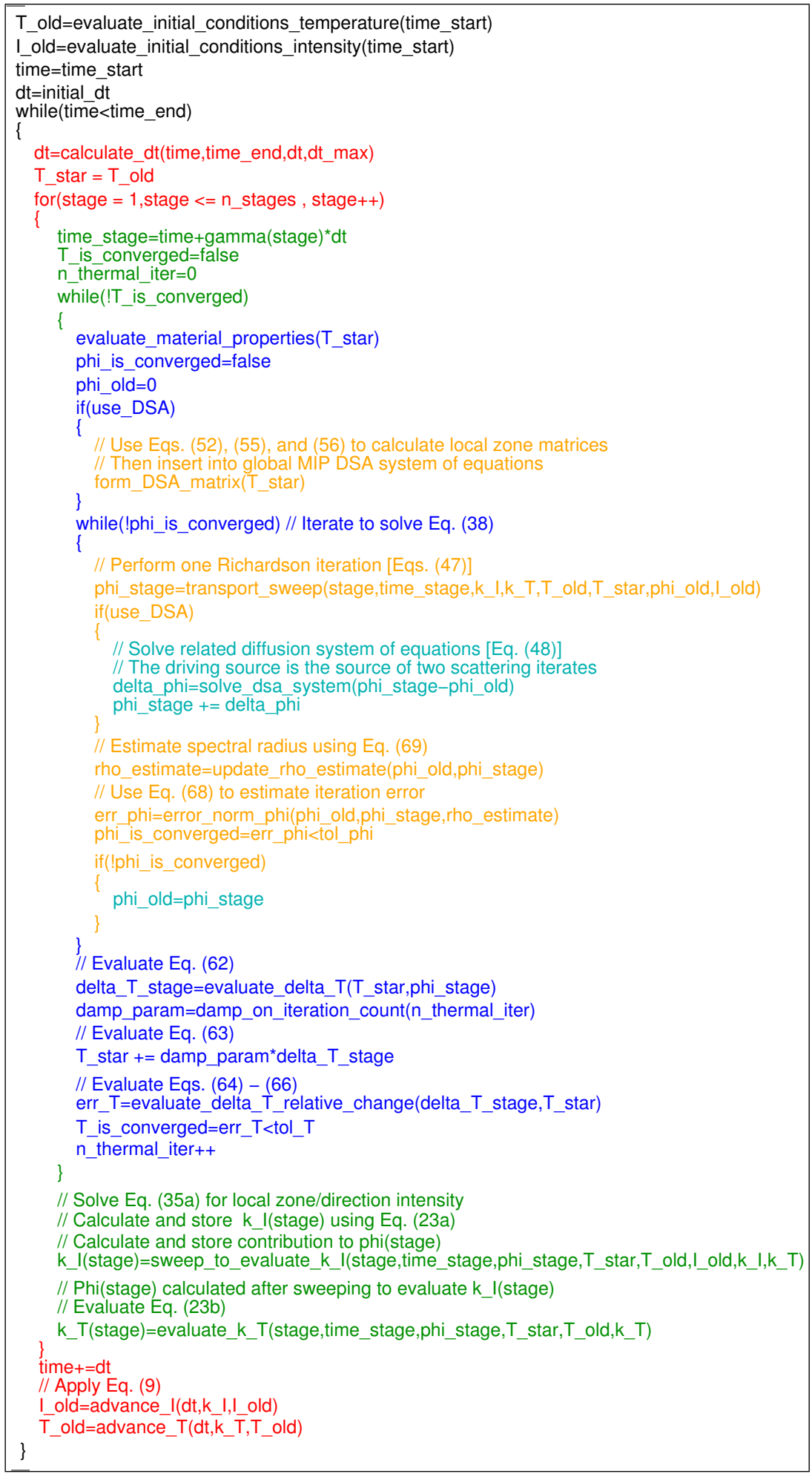

18

Figure 1: Pseudo-code of solution process used to solve grey TRT equations. 
once the thermal iteration has converged. Upon convergence of the thermal iteration at a given time stage we prepare to move to the next time stage. First, we calculate the partial derivative of the spatial approximation of temperature with respect to time at stage $i, \widetilde{k}_{T}^{i}$, in each zone using Eq. (23b). Then, we sweep the entire angular intensity domain, moving zone by zone, direction by direction,

1. calculating the local DFEM approximation of the intensity solution at stage $i, \widetilde{I}_{d, z}^{i}$, using the converged temperature iterate to evaluate material properties,

2. applying the definition of Eq. (23a) to calculate $\vec{k}_{I}^{i}$, and

3. storing $\vec{k}_{I}^{i}$, then moving to the next, downwind mesh cell and repeating.

This iteration scheme requires us to store the following angular intensity related unknowns:

1. the previous time step intensity intensity solution representation, $\widetilde{I}_{d}^{n}$, of size $N_{d i r} \times N_{z} \times N_{P}$ and

2. the intensity solution representation derivative with respect to time at each SDIRK stage, $\widetilde{k}_{I_{d}}^{i}$, of size $N_{s} \times N_{d i r} \times N_{z} \times N_{P}$.

Additionally, two copies of the angle integrated intensity solution representation, $\widetilde{\phi}^{i}$, each of size $N_{z} \times N_{P}$ are required as part of the inner iterative solve and thermal iteration. Using DSA requires the additional storage of the sparse MIP DSA matrix, requiring storage of less than $N_{z} \times 3 \times N_{P} \times N_{P}$ non-zero matrix entries. The exact minimum size of the diffusion matrix is discretization dependent, e.g Gauss vs. Lobatto DFEM interpolation points.

SDIRK time integration and DFEM spatial discretization of material temperature requires storage of $\left(N_{s}+1\right) \times N_{z} \times N_{P}$ temperature related unknowns $\left(T^{n}\right.$ and $\left.k_{T}^{i}\right)$. We store two additional temperature related unknowns, $T^{*}$ and T_reset, as part of our nonlinear thermal iteration scheme, bringing the total amount of material temperature storage to $\left(N_{s}+3\right) \times N_{z} \times N_{P}$ unknowns.

Equation (36) can be thought of as defining an undamped quasi-Newton 
iteration for the temperature in each zone,

$$
\vec{T}^{*,(r+1)}=\vec{T}^{*,(r)}+\vec{\Delta}^{(r)}
$$

where $r$ is a nonlinear iteration index and upon convergence

$$
\vec{T}^{i}=\vec{T}^{*,(r+1)} .
$$

The Newton update, $\vec{\Delta}^{(r)}$, associated with iterate $\vec{T}^{*,(r)}$ in zone $z$ in Eq. (60) is:

$$
\begin{aligned}
\vec{\Delta}^{(r)}=\mathbf{C}^{i}\left[\vec{T}^{n}-\vec{T}^{*}+\Delta t\right. & \left.\sum_{j=1}^{i-1} \alpha_{i j} k_{T}^{j}\right] \\
& +\Delta t \alpha_{i i} \mathbf{C}^{i} \mathbf{R}_{C_{v}}^{-1}\left[\mathbf{R}_{\sigma_{a}}\left(\vec{\phi}^{i}-4 \pi \vec{B}^{*}\right)+\vec{Q}_{T}^{i}\right] .
\end{aligned}
$$

A damped variant of Eq. (60) simply multiplies $\Delta\left(T^{*}\right)$ by damping parameter, $\delta, \delta \in(0,1]$

$$
\vec{T}^{*,(r+1)}=\vec{T}^{*,(r)}+\delta \vec{\Delta}^{(r)} .
$$

We begin each thermal iteration with $\delta=1$, and use the same damping parameter in each zone. For most cases, we observe thermal iteration convergence within approximately 10 iterations, without the need to damp. To stop divergent thermal iterations, we use a simple damping and reset routine based on iteration count. If the thermal iteration count reaches an iteration cutoff, $r_{c}$, with $r_{c}$ typically equal to 10-20 iterations, we

1. multiply $\delta$ by decrease factor $\theta$, typically with $\theta \in[0.3,0.8]$,

2. reset the iteration count, $r=0$,

3. increase $r_{c}, r_{c}=\operatorname{ceil}\left[\frac{r_{c}}{\delta}\right]$, and

4. reset the thermal iteration to the initial temperature iterate of the given time stage, $T^{*,(0)}=$ T_reset .

The above implementation has not failed during our testing. In our experience the choice of $\theta$ is not critical, and is best chosen based on the nonlinear behavior of the problem: 
1. for rapidly divergent iterations, smaller $\theta$ closer to 0.3 are suggested and

2. for problems that initially converge well, but then slowly begin to diverge, choosing $\theta$ closer to 0.8

has performed best in our testing. In practice, the only requirements are that $0<\theta<1$. If a situation arises where the choice of $\theta$ appears to be critical to convergence of the non-linear iteration, rather than expend resources on experimentally finding the "optimal" $\theta$, we would instead recommend more rigorous selection of $\delta$ at each Newton iteration with techniques like those suggested in [25], rather than periodically reducing $\delta$ based on iteration count.

\subsection{Thermal Iteration Convergence Criteria}

During the nonlinear thermal iteration process, we estimate convergence using a relative point-wise change criterion. The nonlinear thermal iteration at each stage is converged when the maximum point-wise change after an iteration, $\varepsilon_{T}^{(r)}$, is less than our convergence tolerance, $\epsilon_{T}$. The maximum point-wise change is defined as the maximum over all zones,

$$
\varepsilon_{T}^{(r)}=\max _{z}\left[\varepsilon_{T, z}^{(r)}\right]
$$

of the zone local maximum change, $\varepsilon_{T, z}^{(r)}$,

$$
\varepsilon_{T, z}^{(r)}=\max _{\ell}\left[\varepsilon_{T, z, \ell}^{(r)}\right] .
$$

The point-wise normalized difference, $\varepsilon_{T, z, \ell}^{(r)}$, at thermal iteration $r$, in zone $z$, at unknown $\ell$, is

$$
\varepsilon_{T, z, \ell}^{(r)}=\left\{\begin{array}{cc}
\left|\frac{\vec{\Delta}_{\ell}^{(r)}}{T_{z, \ell}^{*,(r)}}\right|, & \left|T_{z, \ell}^{*,(r)}\right|>T_{\min } \\
\left|\vec{\Delta}_{\ell}^{(r)}\right|, & \text { otherwise }
\end{array}\right.
$$

where $T_{\min }$ is a small, positive temperature, such as the minimum initial temperature, that gives the magnitude of a near zero magnitude temperature, given the problem's physical scale or dimensions, and $\vec{\Delta}^{(r)}$ is the same $\vec{\Delta}^{(r)}$ of Eq. (62). We use $\vec{\Delta}^{(r)}$ rather than $\vec{T}^{*,(r+1)}-\vec{T}^{*,(r)}$ in Eq. (66) to prevent small damping parameters from indicating premature convergence. 


\subsection{Angle Integrated Intensity Convergence Criteria}

When iterating to find $\phi^{i}$ for a given temperature iterate, the traditional error estimation, $\varepsilon_{\phi}^{(\ell)}$,

$$
\varepsilon_{\phi}^{(\ell)}=\frac{\left\|\phi^{(\ell+1)}-\phi^{(\ell)}\right\|}{\left\|\phi^{(\ell+1)}\right\|},
$$

can lead to false convergence. As shown in [26], false convergence can be eliminated by normalizing Eq. (67) by diving by $1-\rho$,

$$
\varepsilon_{\phi}^{(\ell)}=\frac{\left\|\phi^{(\ell+1)}-\phi^{(\ell)}\right\|}{(1-\rho)\left\|\phi^{(\ell+1)}\right\|}
$$

where $\rho$ is the spectral radius. For $(\ell)>1$, we estimate the spectral radius as:

$$
\rho \approx \frac{\left\|\phi^{(\ell+1)}-\phi^{(\ell)}\right\|}{\left\|\phi^{(\ell)}-\phi^{(\ell-1)}\right\|} .
$$

We use the definition of $\varepsilon_{\phi}$ given in Eq. (68) to terminate the search for $\phi^{i}$ for a given temperature iterate. In this work, we use the $L_{1}$ norm to evaluate all norms related to angle integrated intensity convergence.

\subsection{Possibility of Negative Temperatures}

It has been shown that higher order self-lumping DFEM methods applied to source free pure absorber linear neutron transport problems with spatially varying cross sections do not yield strictly non-negative solutions [10]. As such, we must consider the possibility of negative intensities leading to negative temperature solutions. To accommodate negative temperature solutions, we must define material properties and the Planck emission, for non-physical, negative temperatures. We use the negative temperature definitions defined by Morel et. al [9] for self-adjoint angular intensity forms of the thermal radiative transfer equations and repeat them here for completeness. First, we require that

all temperature-dependent material properties, $\sigma_{a}, \sigma_{s}$, and $C_{v}$ remain positive, despite negative temperature values. In particular, we define $\sigma_{a}$ to be

$$
\sigma_{a}(T)=\left\{\begin{array}{ll}
\sigma_{a}\left(T_{\text {cold }}\right) & T<T_{\text {cold }} \\
\sigma_{a}(T) & \text { otherwise }
\end{array},\right.
$$


where $T_{\text {cold }}$ is a temperature floor, typically the lowest initial material temperature in the problem. Following the arguments of [9], for an arbitrary positive temperature, $\bar{T}$,

$$
\begin{aligned}
B(-\bar{T}) & =-B(\bar{T}) \\
\left.\frac{d B}{d T}\right|_{T=-\bar{T}} & =-\left.\frac{d B}{d T}\right|_{T=\bar{T}} .
\end{aligned}
$$

The above modifications are required for all schemes considered, including SL Lobatto and SL Gauss. Though [10, 18] demonstrated that even degree SL Gauss and odd degree SL Lobatto schemes yielded strictly positive outflow angular fluxes for source free pure absorbers when assuming a zone-wise constant cross section, [18] also demonstrated that a pure absorber with a volumetric source possessing a strong spatial gradient could lead to negative angular flux solutions, despite assuming a zone-wise constant cross section. In thermal radiative transfer, the variation in temperature and resultant variation in Planck function emission creates volumetric sources with strong spatial gradients, leading to the possibility that all schemes and trial space degrees may generate negative angular flux solutions.

\section{Numerical Results}

In this section we present a series of test problems that demonstrate the performance of our high-order SL DFEM schemes for grey thermal radiative transfer. First, we compare solutions from our simulation methodology to the semi-analytic solution of Su and Olson [27]. Then, we consider manufactured solution problems to demonstrate both the temporal and spatial accuracy of our SDIRK time integration and SL DFEM discretization. Finally, we apply our methods to a series of Marshak wave problems, demonstrating that our higher order solution techniques can be applied to practical thermal radiative transfer problems of interest to yield tangible results. 


\subsection{Su-Olson Benchmark}

The Su-Olson benchmark [27] is a grey, time dependent slab geometry thermal radiative transfer problem consisting of an initially cold, half space slab heated by a volumetric source for a set amount of time. By assuming

$$
C_{v} \propto T^{3}
$$

semi-analytic solutions to the grey TRT equations can defined. Since the slab is initially cold, $T=0$, the assumption of Eq. (72) creates numerical difficulties when solving explicitly for material temperature rather than material internal energy. To overcome this difficulty, we use a modified specific heat,

$$
C_{v}=C_{v, o f f}+\eta T^{3}
$$

where $C_{v, \text { of } f}$ is a small offset, and $\eta$ is a chosen coefficient. Though not exactly true to the $\mathrm{Su}-\mathrm{Olson}$ specification, $C_{v, \text { off }} \neq 0$ is required so that when $T=0$, the material can absorb energy. We define the radiation constant and speed of light to be dimensionless, $a=c=1, \sigma_{a}=1 ; \sigma_{s}=0$; place the driving volumetric source in $x \in[0,0.5]$; and choose $\eta=4$ with $C_{v, \text { off }}=10^{-4}$. We truncate the half space to $x \in[0,5]$ since $x=5$ exceeds the maximum distance any particle born in the source could travel, $x_{\max }$. Given our problem parameters, $x_{\max }=0.5+\tau_{\text {end }}$, where $\tau_{\text {end }}$ is the end of the simulation and $\tau_{\text {end }}=3.16228$.

All results use a computational mesh with 400 total spatial zones, 160 zones are equally spaced between $x \in[0,0.5]$, and 240 zones logarithmically spaced between $x \in[0.5,5]$, with smaller zones being placed near $x=0.5$, a $10 \%$ increase in zone width, but a minimum zone width of $\Delta x=0.001$. Additionally, results are generated using an initial time step size of $\Delta t=10^{-4}$, increased by $5 \%$ after each time step, until reaching a maximum time step of $\Delta t=0.01$. We use a Gauss $S_{8}$ angular quadrature.

In Fig. 2 and Fig. 3 we give the radiation energy density solution and material energy density solution, respectively $W(x)$ and $V(x)$ in the notation of Su and Olson [27]. In our notation, $W(x)=\phi(x)$ and $V(x)=[T(x)]^{4}$. Though our computational domain is from $x \in[0,5]$ we only plot $x \in[0,4]$, consistent with 
the data provided in [27], where the largest $x$ data value for $\tau=3.16228$ is given at $x=3.16228$. The results of Fig. 2 are obtained using linear SLXS Lobatto spatial differencing and advanced in time using implicit Euler time integration. Our numerical solutions are compared against the tabulated results in [27] at different dimensionless times, $\tau$, in the notation of [27]. Likewise, the radiation

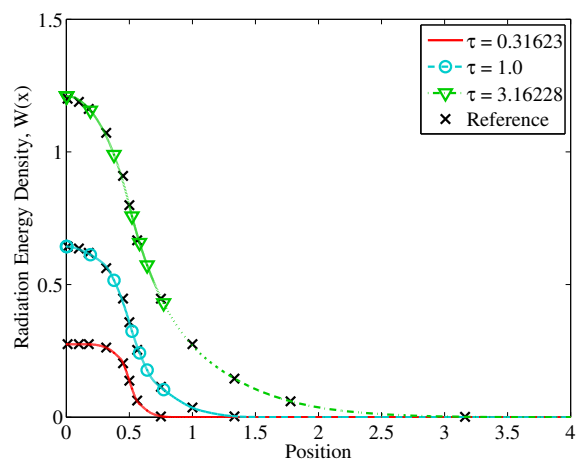

(a) $W(x)=\phi(x)$

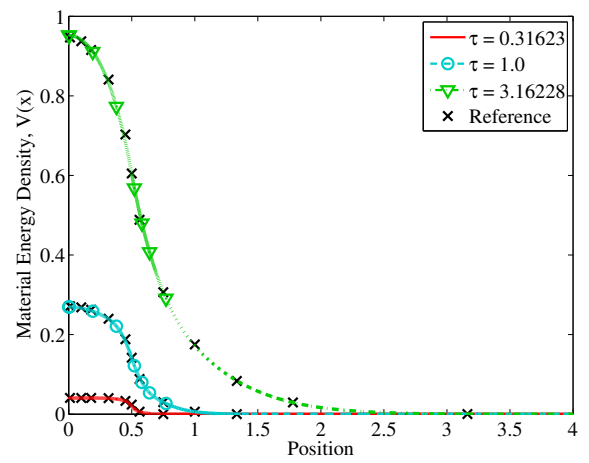

(b) $V(x)=[T(x)]^{4}$

Figure 2: Linear SLXS Lobatto with implicit Euler time integration results for the Su-Olson problem at different times, $\tau$.

energy density and material energy density solutions for a quartic SLXS Gauss discretization are given in Fig. 3. The quartic SLXS Gauss results are integrated in time using the SDIRK 3-3 scheme. The agreement in Fig. 2 and Fig. 3 to the reference solution demonstrates that our SLXS DFEM and SDIRK time integration schemes are solving the grey thermal radiative transfer equations accurately.

\subsection{Asymptotic Orders of Convergence}

We now demonstrate the asymptotic order of convergence of our different DFEM schemes. To asses order of convergence, we use the discrete $L_{2}$ norm of the error in angle integrated intensity, $E_{\phi}=\|\widetilde{\phi}(x)-\phi(x)\|_{L_{2}}$, and the $L_{2}$ error 


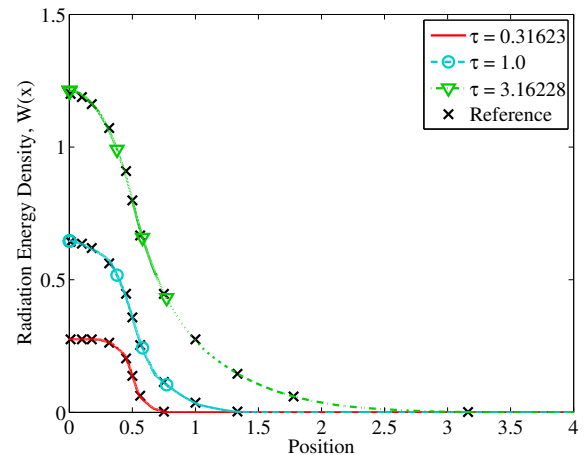

(a) $W(x)=\phi(x)$

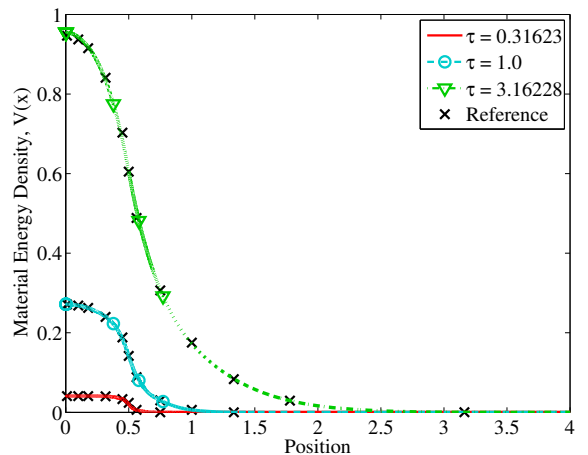

(b) $V(x)=[T(x)]^{4}$

Figure 3: Quartic SLXS Gauss with SDIRK 3-3 time integration results for Su-Olson problem at different times, $\tau$

in temperature, $E_{T}=\|\widetilde{T}(x)-T(x)\|_{L_{2}}$. We calculate $E_{\phi}$ as

$$
E_{\phi}=\sqrt{\sum_{z=1}^{N_{z}} \frac{\Delta x_{z}}{2} \sum_{q=1}^{N_{q f}} w_{q}\left(\widetilde{\phi}\left(s_{q}, t_{\text {end }}\right)-\phi\left(s_{q}, t_{\text {end }}\right)\right)^{2}},
$$

where $w_{q}, s_{q}$ are Gauss quadrature points, we use $N_{q f}=2 P+7$ points to evaluate the spatial error, and $P$ is the DFEM trial space degree. $E_{T}$ is calculated analogously to $E_{\phi}$. Additionally, we will consider $L_{2}$ like norms of the zone average angle integrated intensity, $E_{\phi_{A}}$, and zone average temperature error, $E_{T_{A}} \cdot E_{\phi_{A}}$ is approximated as:

$$
E_{\phi_{A}}=\sqrt{\sum_{z=1}^{N_{z}} \frac{\Delta x_{z}}{2}\left(\frac{1}{2} \sum_{q=1}^{N_{q f}} w_{q} \widetilde{\phi}\left(s_{q}, t_{\text {end }}\right)-\frac{1}{2} \sum_{q=1}^{N_{q f}} w_{q} \phi\left(s_{q}, t_{\text {end }}\right)\right)^{2}} .
$$

$E_{T_{A}}$ is estimated in a similar fashion.

To determine asymptotic order of convergence, we use the method of manufactured solutions (MMS) [28, 29]. With MMS, we define the source necessary to obtain a desired analytic solution. We elect to choose separable manufactured 
solutions of the form:

$$
\begin{aligned}
I_{d}(x, t) & =\mathcal{M}_{d} \mathcal{F}(t) \mathcal{W}_{I}(x) \\
T(x) & =\mathcal{F}(t) \mathcal{W}_{T}(x) \\
\phi(x) & =\mathcal{C}_{M} \mathcal{F}(t) \mathcal{W}_{I}(x) \\
\mathcal{C}_{M} & =\sum_{d=1}^{N_{d i r}} w_{d} \mathcal{M}_{d} .
\end{aligned}
$$

In Eqs. (76), $\mathcal{M}_{d}$ is an angular component of the desired intensity solution, $I_{d}(x, t) ; \mathcal{F}(t)$ is the time component of the manufactured solution, chosen to be the same for the angular intensity, angle integrated intensity, and temperature; $\mathcal{W}_{I}(x)$ is the spatial component of the angular intensity; and $\mathcal{W}_{T}(x)$ is the spatial component of the temperature solution. For all MMS simulations, our initial conditions are

$$
\begin{aligned}
I_{d}\left(x, t_{0}\right) & =\mathcal{M}_{d} \mathcal{W}_{I}(x) \mathcal{F}\left(t_{0}\right) \\
T\left(x, t_{0}\right) & =\mathcal{W}_{T}(x) \mathcal{F}\left(t_{0}\right) \\
\phi\left(x, t_{0}\right) & =\mathcal{C}_{M} \mathcal{W}_{I}(x) \mathcal{F}\left(t_{0}\right) .
\end{aligned}
$$

Likewise, we always use an incident flux intensity boundary condition, for $\mu_{d}>$ 0 :

$$
I_{\text {in }}\left(\mu_{d}, t\right)=\mathcal{M}_{d} \mathcal{W}_{I}\left(x_{1 / 2}\right) \mathcal{F}(t)
$$

and for $\mu_{d}<0$

$$
I_{\text {in }}\left(\mu_{d}, t\right)=\mathcal{M}_{d} \mathcal{W}_{I}\left(x_{N_{z}+1 / 2}\right) \mathcal{F}(t),
$$

where $x_{1 / 2}$ is the left domain boundary and $x_{N_{z}+1 / 2}$ is the right domain boundary. We do not impose, and no boundary conditions are required for the material temperature. We approximately evaluate $\vec{Q}_{I}^{i}$ and $\vec{Q}_{T}^{i}$ using the same $N_{q f}$ point Gauss-Legendre quadrature used to evaluate solution error in Eqs. (74- 75). The MMS driving sources are defined as,

$$
\begin{aligned}
Q_{I_{d}}^{i}\left(x, \mu_{d}, t^{i}\right)=\left.\frac{1}{c} \frac{\partial}{\partial t} I_{d}(x, t)\right|_{t=t^{i}}-\frac{\sigma_{s}}{4 \pi} \phi\left(x, t^{i}\right) \\
\quad-\sigma_{a}\left[T\left(x, t^{i}\right)\right] B\left[T\left(x, t^{i}\right)\right]+\sigma_{t}\left[T\left(x, t^{i}\right)\right] I_{d}\left(x, t^{i}\right)-\mu_{d} \frac{\partial}{\partial x} I_{d}\left(x, t^{i}\right)
\end{aligned}
$$




$$
\begin{aligned}
Q_{T}^{i}\left(x, t^{i}\right)=C_{v}\left[T\left(x, t^{i}\right)\right] \frac{\partial}{\partial t} & \left.T(x, t)\right|_{t=t^{i}} \\
& -\sigma_{a}\left[T\left(x, t^{i}\right)\right]\left\{\phi\left(x, t^{i}\right)-4 \pi B\left[T\left(x, t^{i}\right)\right]\right\} .
\end{aligned}
$$

We do not approximate the spatial dependence of the Planck function when evaluating the driving sources, $Q_{I_{d}}^{i}$ and $Q_{T}^{i}$.

\subsubsection{Constant in Space}

We now consider a MMS problem that is constant in space to verify that applying higher order SDIRK time integration strategies to the grey TRT equations leads to higher order temporal accuracy. For this problem, we define our MMS as:

$$
\begin{aligned}
\mathcal{M}_{d} & =\frac{1}{4 \pi} \\
\mathcal{W}_{I}(x) & =\frac{10}{4 \pi} \\
\mathcal{W}_{T}(x) & =10 \\
\mathcal{F}(t) & =10 \exp (-5 t),
\end{aligned}
$$

$t \in[0,1], \sigma_{s}=0.1, \sigma_{a}=2.5, C_{v}=0.2, x \in[0,10]$ discretized with 10 equally spaced zones. Convergence of $E_{\phi}$ and $E_{t}$ as a function of $\Delta t$ for the implicit Euler, SDIRK 2-2, and SDIRK 3-3 time differencing schemes is given in Fig. 4. Solutions are obtained using a constant time step size for this study. As expected, implicit Euler converges first order in time, SDIRK 2-2 converges second order, and SDIRK 3-3 converges third order in time.

\subsubsection{Constant in Time}

To demonstrate the spatial orders of convergence exhibited by the different self-lumping DFEM spatial discretizations, we now consider an MMS problem that is constant in time, but with spatially varying intensity and temperature solutions and temperature dependent material properties. We impose solutions 


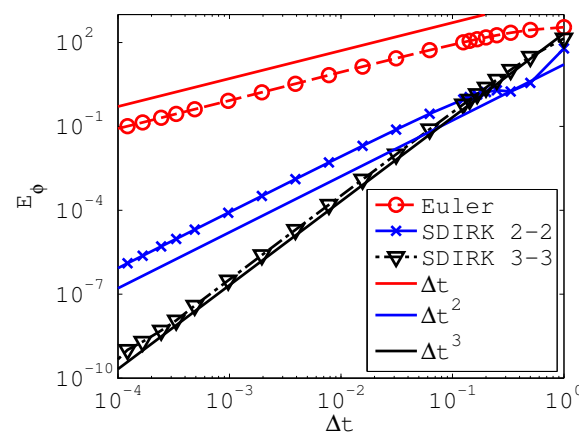

(a) Convergence of $E_{\phi}$

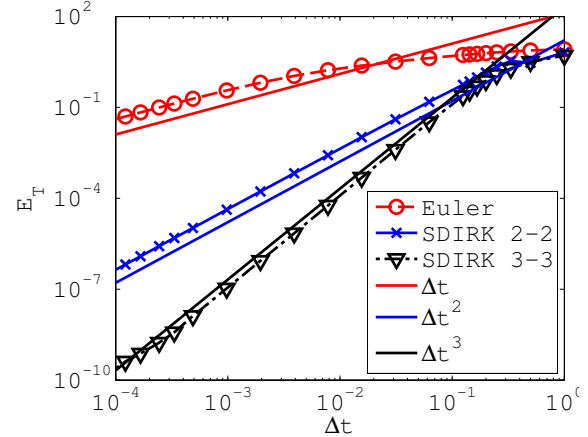

(b) Convergence of $E_{T}$

Figure 4: Temporal convergence for different time integrators as a function of $\Delta t$.

of the form:

$$
\begin{aligned}
\mathcal{M}_{d} & =\frac{1}{4 \pi} \\
\mathcal{W}_{I}(x) & =199 \cos \left(\frac{\pi x}{10}\right)+200 \\
\mathcal{W}_{T}(x) & =190 \cos \left(\frac{\pi x}{10}\right)+200 \\
\mathcal{F}(t) & =10
\end{aligned}
$$

with the following material properties:

$$
\begin{aligned}
C_{v} & =0.1+0.2 T^{2} \\
\sigma_{a} & =\frac{5}{T^{2}} \\
\sigma_{s} & =0.01,
\end{aligned}
$$

for $x \in[0,10], S_{8}$ Gauss quadrature, $t \in[0,1]$ with a constant $\Delta t=0.1$, and implicit Euler time differencing.

$E_{\phi}$ and $E_{T}$ convergence for the SL Lobatto scheme is given in Fig. 5. SL Gauss convergence for the same quantities is given in Fig. 6. Convergence data in Figs. 5-6, and all subsequent convergence plots, is given as function of average zone width, and DFEM trial space degree. Regardless of DFEM interpolation point type or trial space degree, assuming zone-wise constant material properties limits spatial convergence of $E_{\phi}$ to at most second order for problems with 


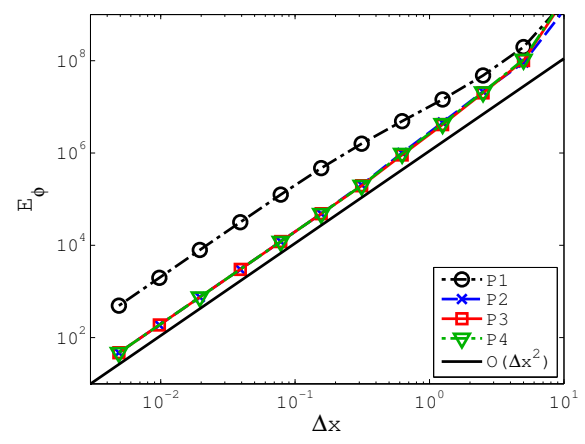

(a) $E_{\phi}$ convergence

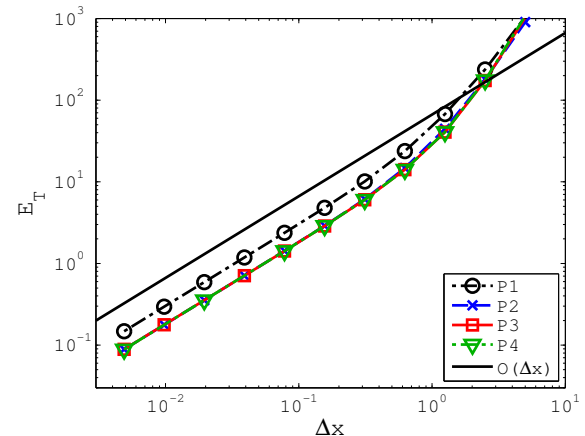

(b) $E_{T}$ convergence

Figure 5: SL Lobatto convergence for the constant in time MMS problem.

spatially varying material properties. Likewise, assuming zone-wise constant material properties for problems with spatially varying material properties limits $L_{2}$ convergence of the temperature to at most first order in space, regardless of DFEM interpolation point type or trial space degree.

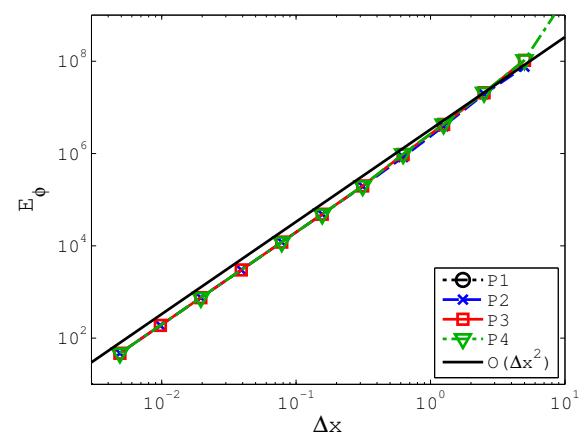

(a) $E_{\phi}$ convergence

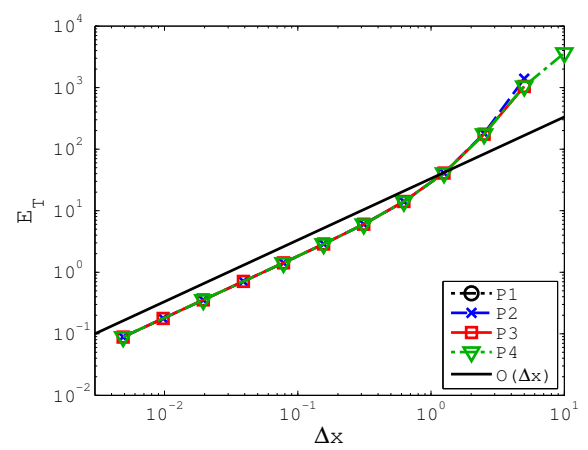

(b) $E_{T}$ convergence

Figure 6: SL Gauss convergence for the constant in time MMS problem.

In [10], for pure absorber neutron transport problems, assuming a zone-wise constant cross section for problems with a spatially varying cross section resulted in convergence of the $L_{2}$ norm of the angular flux error that was second order in space and the error in the point-wise interaction rate converged first order in space, regardless of DFEM trial space degree. Despite this poor $L_{2}$ error 
convergence, schemes that assumed a zone-wise constant cross section for spatially varying cross section pure absorber problems in [10] converged a measure of the zone average interaction rate proportional to $\Delta x^{2 P+1}$. To demonstrate that a similar phenomena does not occur for zone averaged quantities in thermal radiative transfer problems, we plot the convergence of $E_{\phi_{A}}$ and $E_{T_{A}}$ for the SL Gauss scheme in Fig. 7. Regardless of DFEM trial space degree, the SL Gauss

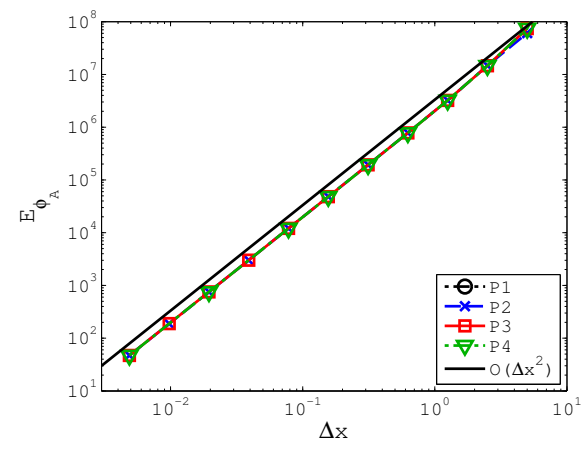

(a) $E_{\phi_{A}}$ convergence

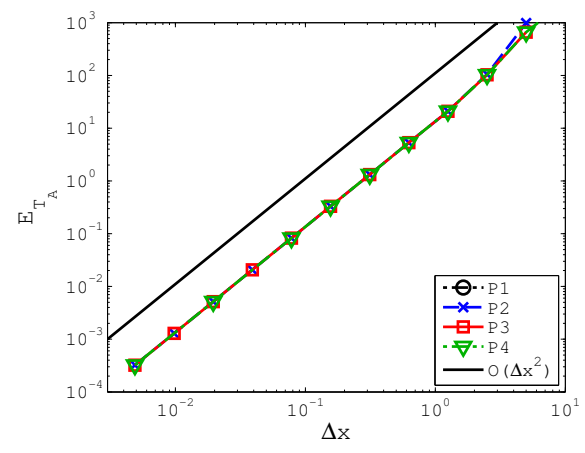

(b) $E_{T_{A}}$ convergence

Figure 7: SL Gauss convergence of zone average quantities for the constant in time MMS problem.

scheme converges $E_{\phi_{A}}$ and $E_{T_{A}}$ at most second order in space for problems with spatially varying material properties. The SL Lobatto scheme exhibits similar zone average quantity convergence behavior as SL Gauss: second order convergence regardless of trial space degree, but we omit this data for brevity.

For thermal radiative transfer problems, the SLXS Lobatto and SLXS Gauss schemes demonstrate increased order of convergence for $E_{\phi}$ and, $E_{T}$, with increases in DFEM trial space degree. However, in our testing, accurate observations of higher order SLXS Gauss convergence rates was challenging and problem dependent. Consider Fig. 8 that gives the convergence of $E_{\phi}$ for the SLXS Gauss scheme for the steady state MMS problem described by Eqs. (85a). In Fig. 8, SLXS Gauss appears to converge $E_{\phi}$ proportional to $\Delta x^{P+2}$, a full order higher than the observed $L_{2}$ error convergence of SLXS Gauss applied to linear neutron transport problems [10]. Only the lower order methods, at very fine 


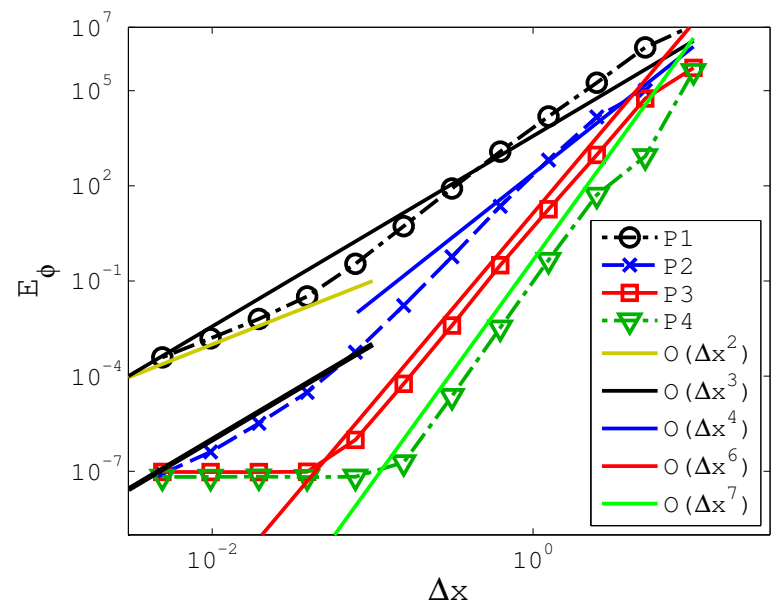

Figure 8: Steady state, cosine spatial dependence SLXS Gauss convergence of $E_{\phi}$.

mesh refinements appear to begin converging $E_{\phi}$ proportional to $\Delta x^{P+1}$. We suspect the cubic and quartic schemes reach the limit of our nonlinear thermal iteration tolerance at coarser meshes (the plateauing of errors) before exhibiting $E_{\phi}$ convergence proportional to $\Delta x^{P+1}$.

If we instead consider a MMS problem of the form:

$$
\begin{aligned}
\mathcal{M}_{d} & =\frac{1}{4 \pi} \\
\mathcal{W}_{I}(x) & =10 \exp [-5 x]+1 \\
\mathcal{W}_{T}(x) & =10 \exp [-6 x]+1, \\
\mathcal{F}(t) & =1
\end{aligned}
$$

with identical material properties, $x \in[0,1], S_{8}$ Gauss quadrature, $t \in[0,1]$ with a constant $\Delta t=0.1$, and implicit Euler time differencing, we observe SLXS Gauss convergence of $E_{\phi}$ proportional to $\Delta x^{P+1}$, as seen in Fig. 9a. SLXS Gauss convergence of $E_{T}$ is given in Fig. $9 \mathrm{~b}$ for the MMS described by Eqs. (86). In both of our steady-state MMS problems, SLXS Gauss is observed to converge $E_{T}$ proportionally to $\Delta x^{P+1}$.

Convergence of $E_{\phi}$ and $E_{T}$ for the SLXS Lobatto scheme for the steady-state MMS described by Eqs. (86) is given in Fig. 10. The observed convergence of 


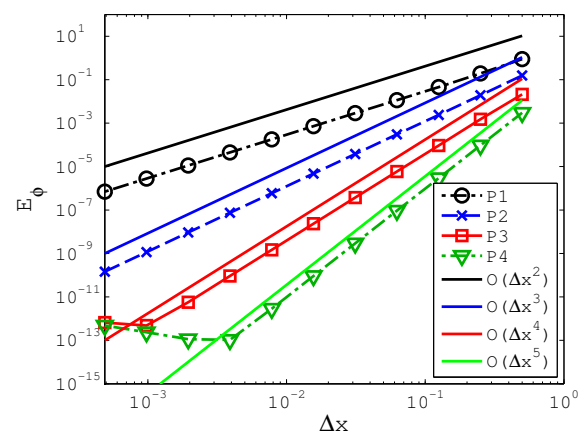

(a) $E_{\phi}$ convergence

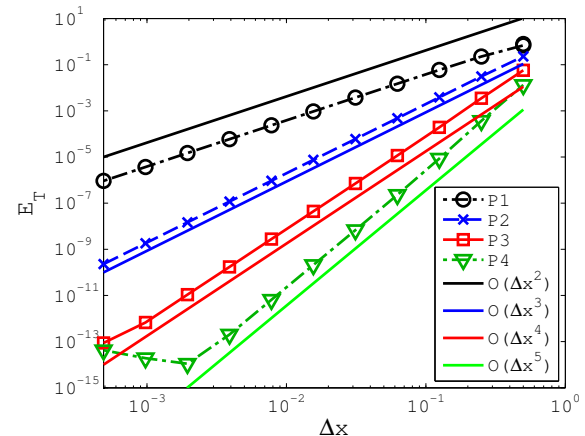

(b) $E_{T}$ convergence

Figure 9: SLXS Gauss convergence rates for a steady state problem with an exponential spatial dependence.

$E_{\phi}$ proportional to $\Delta x^{P+1}$ and $E_{T}$ proportional to $\Delta x^{P}$ in Fig. 10 are identical to the rates observed for the steady state MMS problem described by Eqs. (85a).

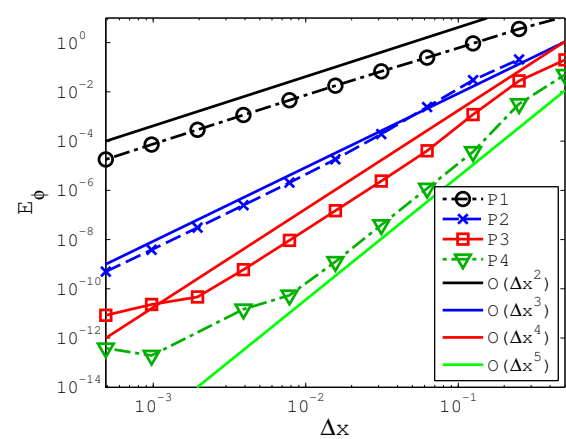

(a) $E_{\phi}$ convergence

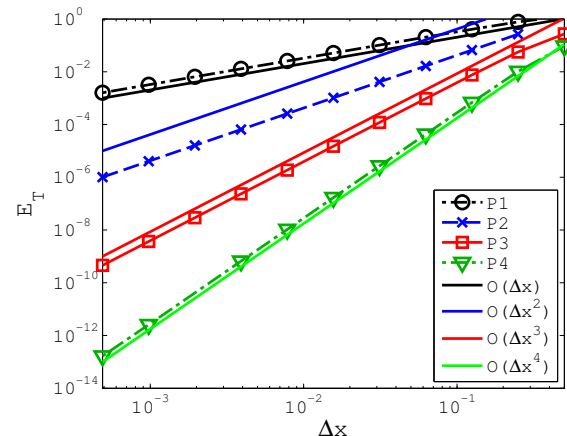

(b) $E_{T}$ convergence

Figure 10: SLXS Lobatto convergence for the steady-state MMS problem with exponential spatial dependence.

Further investigation is required to explain the observed SLXS Lobatto and SLXS Gauss convergence rates. For linear neutron transport, the $L_{2}$ error of scalar flux is observed to converge proportional to $\Delta x^{P+1}$ with both the SLXS Lobatto and SLXS Gauss schemes. Thus, the sub-optimal $E_{T}$ convergence 
of the SLXS Lobatto and possibly greater than expected $E_{\phi}$ convergence of the SLXS Gauss scheme require further investigation. We suspect the possible convergence of $E_{\phi}$ proportional to $\Delta x^{P+2}$ exhibited by by the SLXS Gauss scheme is dependent on the choice of MMS solution form, but further testing is required to confirm this hypothesis. From the results of $[10,18]$, we expect SLXS Gauss to be more accurate than SLXS Lobatto by a constant. The observation that SLXS Lobatto converges $E_{T}$ proportional to $\Delta^{P}$, a full order less than SLXS Gauss, is unexpected. One possible explanation is that the SLXS Gauss scheme is able to exactly integrate $\int b_{\ell}(s) \widetilde{B}_{i, z}(s)$, whereas SLXS Lobatto cannot. Inexact integration of the mass matrix in $[10,18]$ did not lower the SLXS Lobatto scheme's order of convergence relative to SLXS Gauss. However, the grey TRT are fundamentally driven by linearized sources composed of several integrations that SLXS Lobatto approximates less accurately than SLXS Gauss. Thus it may be reasonable that SLXS Lobatto converges $E_{T}$ a full order less than SLXS Gauss. Alternatively, since Gauss quadrature is two orders more accurate than Lobatto quadrature with an equal number of points, SLXS Gauss may achieve $E_{T}$ convergence proportional to $\Delta x^{P+1}$ because the SLXS Gauss approximation of the Planck function spatial dependence is more accurate than the SLXS Lobatto approximation of the Planck function spatial dependence.

\subsubsection{Linear in Time}

To verify that the orders of convergence observed in the steady state problem are not limited to steady state problems, we apply the SLXS Lobatto and SLXS Gauss schemes to a problem that is linear in time. We use the same MMS and material properties described in Eqs. (86), but impose a time dependence of

$$
\mathcal{F}(t)=1+0.05 t
$$

and integrate in time using the Alexander 2-2 scheme (see Appendix A) with a constant time step size, $\Delta t=0.01$. In Fig. 11 , we confirm the steady state results. SLXS Lobatto converges $E_{\phi}$ proportional to $\Delta x^{P+1}$ and $E_{T}$ proportional to $\Delta x^{P}$. SLXS Gauss converges $E_{\phi}$ proportional to $\Delta x^{P+1}$ and $E_{T}$ proportional 
to $\Delta x^{P+1}$.

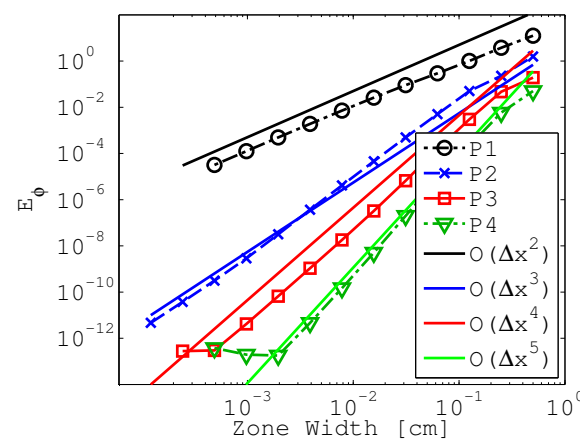

(a) SLXS Lobatto $E_{\phi}$ convergence

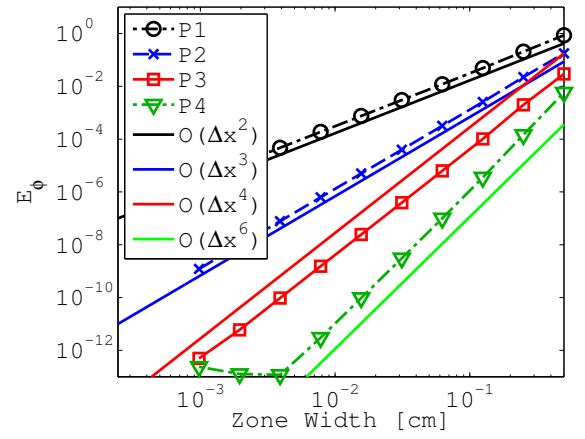

(c) SLXS Gauss $E_{\phi}$ convergence

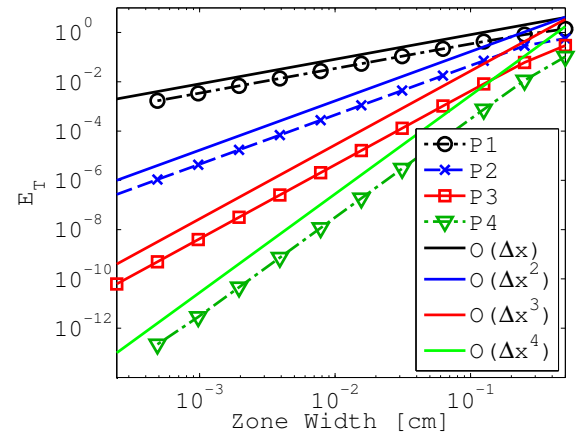

(b) SLXS Lobatto $E_{T}$ convergence

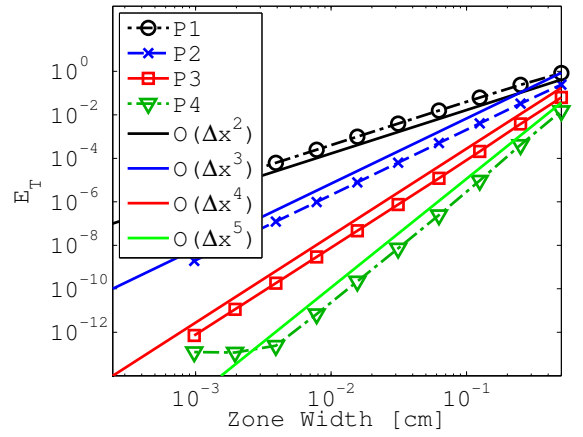

(d) SLXS Gauss $E_{T}$ convergence

Figure 11: SLXS Lobatto and Gauss convergence for the linear in time MMS problem.

In Figs. 8-11, the plateauing of errors that appears in some plots is a result of our nonlinear temperature iteration convergence criteria. To demonstrate this, consider the SLXS Gauss convergence of $E_{T}$ in Fig. 12 after relaxing our convergence tolerance from $\epsilon_{T}=10^{-11}$ and $\epsilon_{\phi}=10^{-13}$ to $\epsilon_{T}=10^{-8}$ and $\epsilon_{\phi}=$ $10^{-10}$ for the linear in time problem. Comparing the $E_{T}$ error plateau of Fig. 12 (on the order of $10^{-10}$ ) to the error plateau of Fig. 11d (on the order of $10^{-13}$ ), we see a roughly 1000-fold difference, consistent with our change in tolerance. Both errors are consistent with an expected minimum error achievable, $E_{T, \min }$, given our chosen convergence criteria,

$$
E_{T, \text { min }}=\int_{x_{1 / 2}}^{x_{N_{z}+1 / 2}} \epsilon_{T}\left[\mathcal{F}_{t}\left(t_{\text {end }}\right) \mathcal{W}_{T}(x)\right] d x .
$$




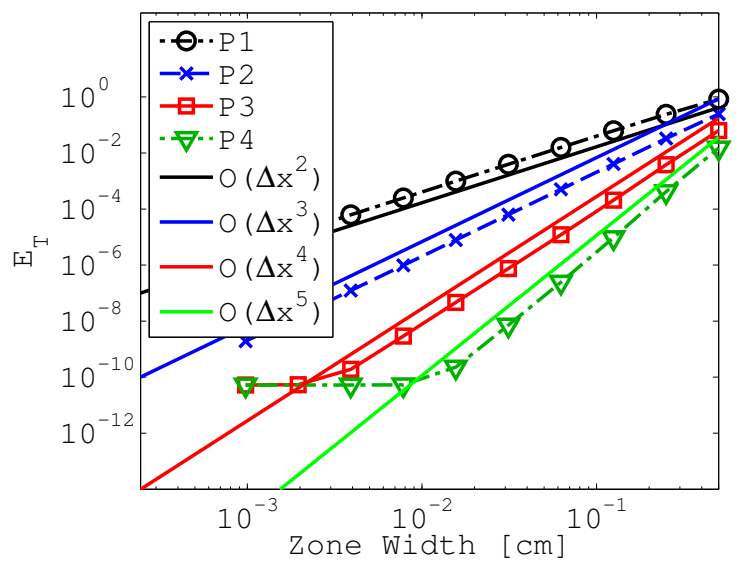

Figure 12: Convergence of $E_{T}$ for the SLXS Gauss scheme for the linear in time MMS problem using $\epsilon_{T}=10^{-8}$ and $\epsilon_{\phi}=10^{-10}$.

\section{3. $S_{2}$ Variant of a Radiation Diffusion Marshak Wave Test Problem}

We now consider a Marshak wave test problem adapted from the radiation diffusion problem given in [7]. As in [7], we arbitrarily assume $a=c=C_{v}=1$. The physical domain is $x \in[0,1]$ and we advance the solution from $t=0$ to $t=1$. Initially, the slab is in thermal equilibrium, and $T=\left(10^{-5}\right)^{1 / 4}$. There is no scattering and the absorption opacity is temperature dependent, $\sigma_{a}=\frac{1}{T^{3}}$. We adapt the radiative diffusion problem to a discrete ordinates problem by using $S_{2}$ Gauss angular quadrature with a unit incident current on the left boundary and a vacuum boundary condition of the right side of the slab. Our initial results use 100 equally spaced spatial zones, linear DFEM, SDIRK 2-2 time integration, an initial time step of $\Delta t=10^{-4}$, and a maximum time step of $\Delta t=10^{-3}$. Time step sizes are increased by $5 \%$ after each time step until the maximum time step size is reached. In the plots that follow, only $x \in[0,0.5]$ is plotted due to the solution for $x \in[0.5,1]$ essentially remaining constant (at the initial conditions) for $t \leq 1$.

The $t=1.0$ radiation profiles for the SL Lobatto and SLXS Lobatto schemes scheme are given in Fig. 13a with corresponding temperature profiles given in Fig. 13b. The linear discontinuous SL Lobatto spatial scheme is equivalent to 


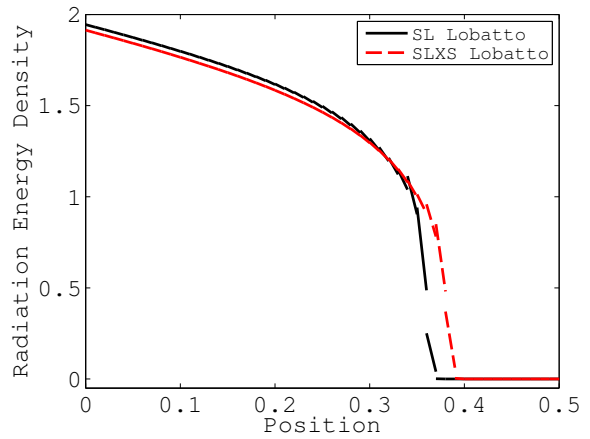

(a) Angle integrated intensity

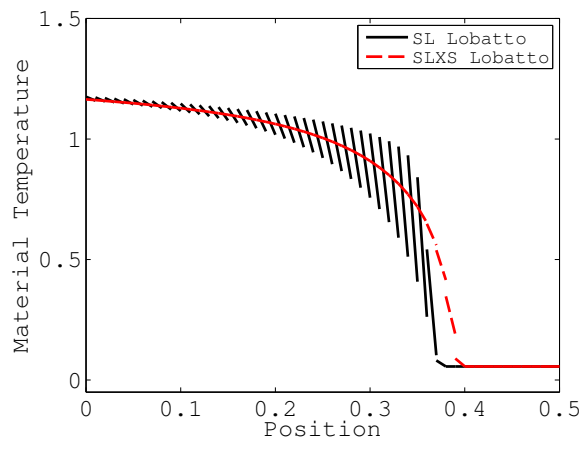

(b) Material temperature

Figure 13: Linear SL Lobatto and SLXS Lobatto Marshak wave solutions at $t=1.0$, using 100 equally spaced zones.

the traditional lumped linear DFEM with zone-wise constant material properties $[10,18]$. Without apriori knowledge of the solution, both solutions in Fig. 13a appear reasonable, albeit spatially under resolved. However, the SL Lobatto temperature profile in Fig. 13b is clearly incorrect; the SL Lobatto temperature profile exhibits large, non-monotonic discontinuities, or blading [10].

The blading phenomena is a non-physical artifact caused by assuming zonewise constant opacities when opacity is a spatially varying quantity. In the Marshak wave problem, opacity is lower where the material is hotter [towards the left boundary] and opacity is greater in colder regions [towards the right boundary]. Thus, a zone-wise constant volumetric average opacity is larger than the opacity on the left edge of a zone and smaller than the opacity on the right edge of the zone for a Marshak wave problem with incident photons on the left edge and vacuum boundary conditions on the right. Since photon energy deposition is proportional to absorption opacity, the zone-wise constant cross section of the SL Lobatto and SL Gauss schemes results in over prediction of photon energy deposition near the hotter, left edge of the zone, and under deposition of photon energy on the right side of the zone. This results in a temperature on the left side of the zone that is too high and a temperature on the right side of the zone that is too low. The temperature within a zone eventually 
equilibrates as the hotter left edge radiates more thermal energy than the right edge radiates, as seen in Fig. 13b; the material nearest the left boundary has been hot the longest, has had the most amount of time to equilibrate, and thus the blading within zones near the left edge is smaller than zones nearer the Marshak wavefront. The SLXS Lobatto and SLXS Gauss schemes do not exhibit the blading phenomena because they use different opacities for different spatial points within a zone, resulting in a much smoother, more physical temperature profile.

The presence of the blading phenomena in a discontinuous temperature profile is not unique to our work. Historically though, only the zone average temperature is plotted, not the full discontinuous temperature profile. Plotting and connecting the mid point [zone average temperature] of the discontinuous SL Lobatto temperature solution of Fig. 13b, yields a qualitatively pleasing temperature solution that is visually close to the discontinuous SLXS Lobatto temperature solution, as shown in Fig. 14. While plotting a piecewise linear in-

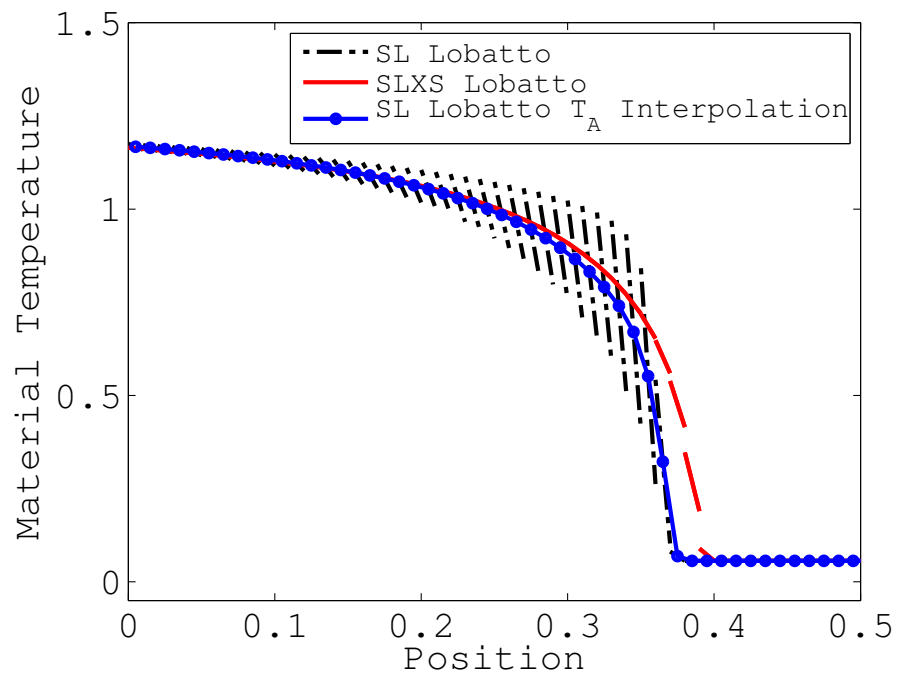

Figure 14: SL Lobatto discontinuous temperature profile and a piecewise linear interpolation of the SL Lobatto zone average temperatures compared to the SLXS Lobatto discontinuous temperature profile.

terpolation of the SL Lobatto zone average temperature makes the SL Lobatto 
solution appear acceptable, it is important to note that the interpolated SL Lobatto thermal wave front location is visibly different than the more accurate thermal wave front position predicted in the SLXS Lobatto solution.

In Fig. 15, we demonstrate that the blading phenomena is solely caused by assuming zone-wise constant material properties. To maximize accuracy for a given number of unknowns, the plots of Fig. 15 use unequally spaced meshes, preferentially placing zones where the solution is changing and minimizing the number of cells used to resolve the region where the Marshak wave does not reach for $t \leq 1$, as described in Table 1. As in Fig. 13, we use linear SL Lobatto,

\begin{tabular}{|c|c|c|}
\hline \multirow{2}{*}{ Legend Entry } & \multicolumn{2}{|c|}{ Equally Spaced Zones } \\
\cline { 2 - 3 } & $x \in[0,0.4]$ & $x \in[0.4,1.0]$ \\
\hline $50 Z$ & 45 & 5 \\
\hline $125 \mathrm{Z}$ & 115 & 10 \\
\hline $250 \mathrm{Z}$ & 235 & 15 \\
\hline $1000 \mathrm{Z}$ & 950 & 50 \\
\hline
\end{tabular}

Table 1: Mesh spacing used in Figs. 15b-17d.

unless otherwise noted, SDIRK 2-2 time integration with an initial time step of $\Delta t=10^{-4}$ and a maximum time step of $\Delta t=10^{-3}$. All results are shown for $t=1.0$. Figure 15a demonstrates that mesh refinement reduces the magnitude of the blading phenomena, but does not eliminate the blading phenomena associated with assuming a zone-wise constant opacity when opacity is a spatially varying quantity. Likewise, Fig. 15b shows that increasing the DFEM trial space degree does nothing to eliminate the blading phenomena. Additionally, Fig. 15b demonstrates that while the modified definitions of opacity, Planck emission, and Planck derivative permit the nonlinear iteration process to proceed despite encountering negative temperatures, negative temperature solutions still indicate that the spatial mesh must be refined to yield accurate solutions. Finally, we show that blading is not limited to the SL Lobatto scheme or odd degree DFEM trial spaces, as demonstrated by the quadratic SL Gauss 
temperature profile in Fig. 15c. In Fig. 15c, the SL Gauss solution appears to indicate a range of temperatures in each zone, however, this is only an artifact of the plot line width. A similar visualization artifact is present in the SL Lobatto solutions of Fig. 16 when using 250 and 1000 spatial zones.

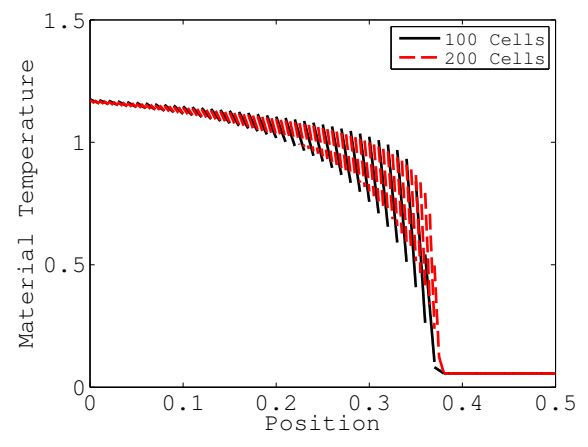

(a) Mesh refinement

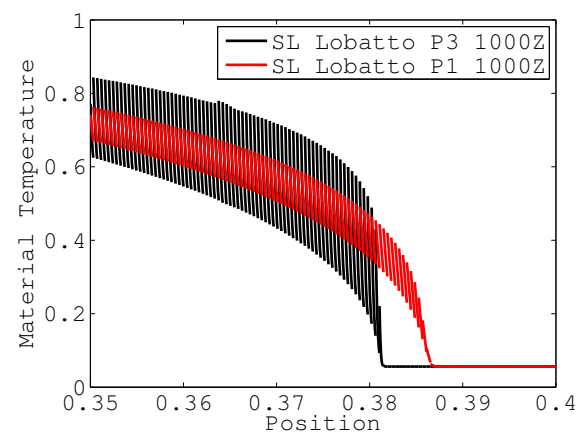

(b) Higher trial space degree

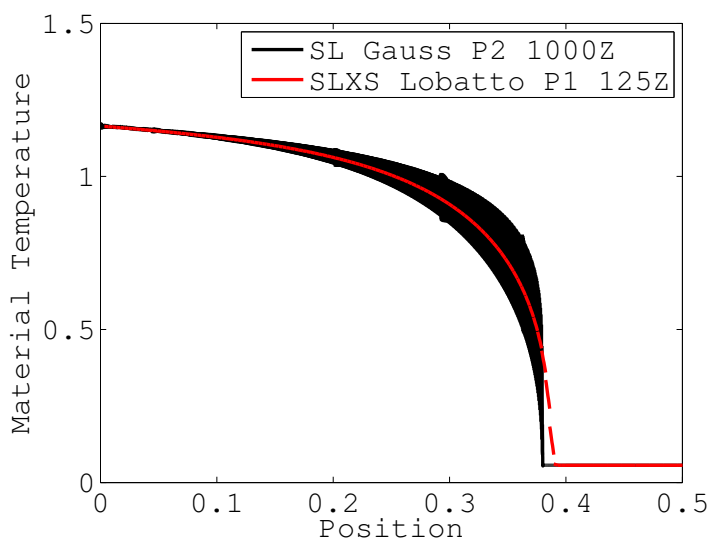

(c) Gauss interpolation points

Figure 15: Changing discretization parameters does not eliminate the temperature profile blading associated with the zone-wise constant material property assumption of the SL Lobatto and SL Gauss schemes.

The negativities and inaccuracy associated with spatially under resolved higher order DFEM solutions are highlighted by Fig. 16. For reference purposes, we compare the cubic SL Lobatto solutions of Fig. 16 to a lower resolution linear SLXS Lobatto solution. The inaccuracy of the cubic SL Lobatto 


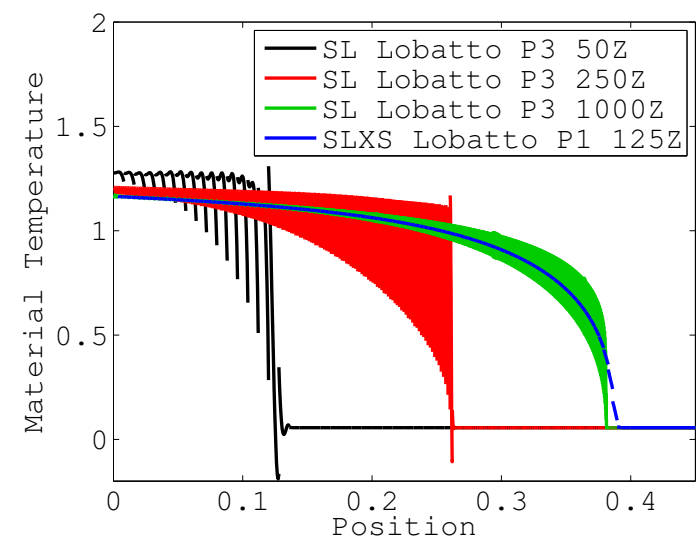

Figure 16: Modifications allow the nonlinear iteration to continue with negative solutions, but large magnitude negative solutions [more prevalent at lower resolutions], greatly affect simulation results.

solution at coarse resolutions is clear; the position of the thermal wavefront moves considerably with mesh refinement.

For completeness, we give the temperature profiles of the cubic SLXS Lobatto solution at varying spatial resolutions and a quadratic SLXS Gauss temperature profile in Fig. 17. Both higher order schemes are compared to a linear SLXS Lobatto solution that uses 125 zones. In Fig. 17a and Fig. 17b, there is little visual difference between the SLXS solutions. However, if we zoom in near the thermal wavefront as in Fig. 17c and Fig. 17d, we observe that the higher resolution and higher order schemes reveal finer structure not present in the lower resolution solutions. Though the choice of DFEM trial space degree, DFEM interpolation point type, and mesh resolution affects the severity of the blading phenomena, the temperature profiles generated by the SLXS Lobatto and SLXS Gauss schemes shown in Fig. 17 allow us to conclude that the temperature profile blading of SL Gauss and SL Lobatto is solely a consequence of assuming a zone-wise constant opacity for problems with spatially varying opacity. 


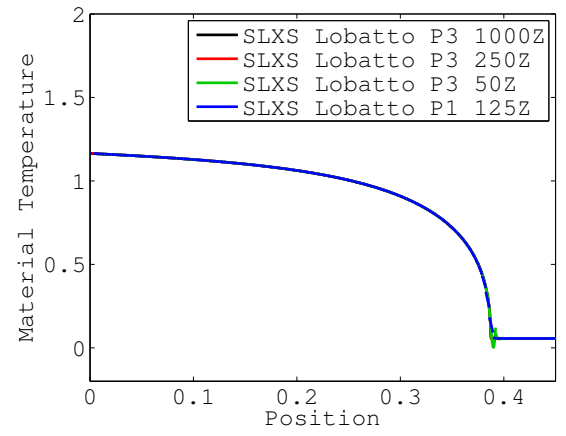

(a) Cubic SLXS Lobatto

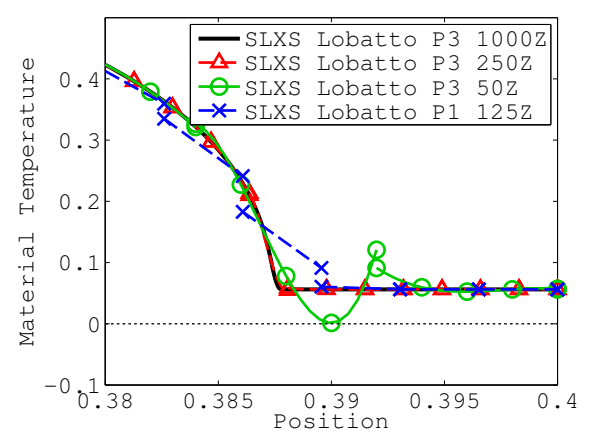

(c) Cubic SLXS Lobatto zoom

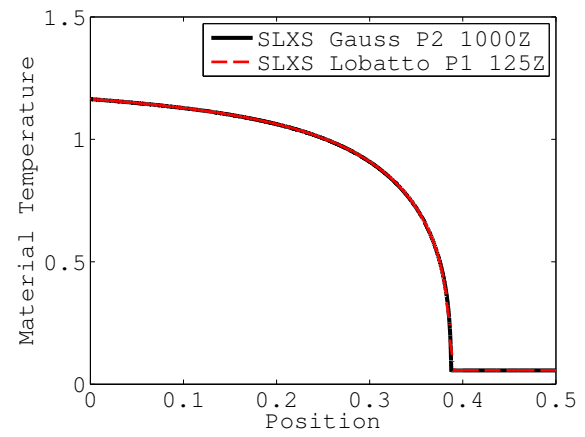

(b) Quadratic SLXS Gauss

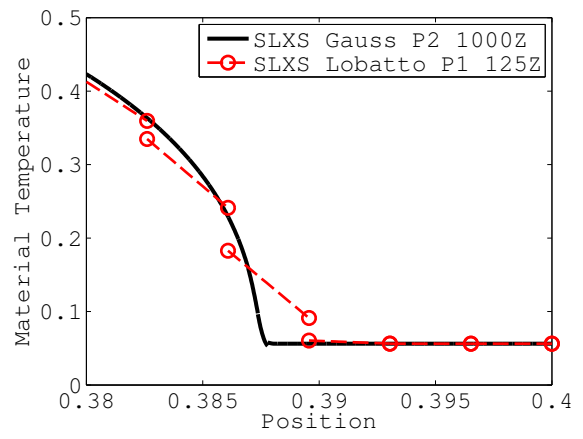

(d) Quadratic SLXS Gauss zoom

Figure 17: Higher order SLXS Lobatto and Gauss temperature solutions for the Marshak wave problem at $t=1.0$. 


\subsection{Effectiveness of Diffusion Synthetic Acceleration}

To demonstrate the iterative effectiveness of MIP DSA we now present a Marshak wave like problem designed to be optically thick and diffusive. Initially, a slab is in thermodynamic equilibrium at a temperature of $T=0.5$, and is heated with an incident current of 100 on the left hand side of the slab. The problem domain is $x \in[0,100], t \in[0,5]$, and for convenience, we define $a=c=$ 1. The slab has a constant heat capacity, $C_{v}=0.05$, does not scatter photons, $\sigma_{s}=0$, and has a temperature dependent absorption opacity, $\sigma_{a}=\frac{5000}{T^{2}}$.

In cases where $\sigma_{a}$ is proportional to $\frac{1}{T^{3}}$ the eigenvalues of $S^{i}$ are not near unity. As an example, consider Eq. (59) when $T_{z, 1}^{*}=T_{z, 2}^{*}=\bar{T}, \sigma_{s}=0, \sigma_{a}=\frac{1}{T^{3}}$, and $C_{v} \rightarrow 0$. In this case, Eq. (59) is:

$$
\begin{gathered}
\mathbf{S}^{i} \approx \frac{c \Delta t \bar{T}^{3}}{\bar{T}^{3}+c \Delta t}\left[\begin{array}{cc}
\frac{1}{\bar{T}^{3}}\left(4 \pi \Delta t \frac{1}{\bar{T}^{3}} \frac{4 \bar{T}^{3}}{4 \pi}\right) & 0 \\
0 & \frac{1}{\bar{T}^{3}}\left(4 \pi \Delta t \frac{1}{\bar{T}^{3}} \frac{4 \bar{T}^{3}}{4 \pi}\right)
\end{array}\right] \\
\mathbf{S}^{i}=\frac{c \Delta t}{\bar{T}^{3}+c \Delta t}\left[\begin{array}{cc}
4 \Delta t & 0 \\
0 & 4 \Delta t
\end{array}\right] .
\end{gathered}
$$

Given Eqs. (89), for every test problem thus far considered, source iteration alone has results in a nearly equivalent iteration count compared to using source iteration combined with MIP DSA. This motivates us to choose $\sigma_{a} \propto \frac{1}{T^{2}}$ for this particular test problem to ensure that as the problem heats up, $\overline{\bar{\nu}}_{i} \rightarrow 1$.

Discretizing this problem with linear SLXS Lobatto using 50 spatial cells, implicit Euler time integration, and a maximum time step size of $\Delta t_{\max }=0.1$ and solving with source iteration alone versus source iteration combined with MIP DSA yields the total number of transport iterations for problem completion given in Table 2.

Noting that both methods used the same number of thermal iterations, it is clear that MIP DSA is an effective iterative acceleration technique for grey thermal radiative transfer simulations. 
Table 2: Iteration count for the optically thick and diffusive TRT problem. Total number of intensity iteration used over all 125 time steps.

\begin{tabular}{|c|c|}
\hline Intensity Iterative Strategy & Total Number of Intensity Iterations (Sweeps) \\
\hline SI & $1,740,320$ \\
\hline SI+DSA & 1,348 \\
\hline
\end{tabular}

\subsection{Computational Performance}

We omit computational timing results from this paper, despite conducting a timing analysis of the code used to generate the results of this manuscript. In our timing study, for a fixed number of unknowns, using higher order methods actually resulted in reduced simulation wall clock time. Our observed trends should not be interpreted as refuting the results of more performance oriented code projects $[3,4,5,30]$. On current computing hardware, higher order methods will almost always incur a time to solution penalty relative to lower order methods that require fewer FLOPs per unknown, as shown in the following thought experiment. For a fixed number of unknowns, $N_{u n k}$, we assume that

1. the intensity update dominates solution time, as is widely accepted within the radiative transfer community,

2. in an optimized code, the MIP DSA contribution to overall time to solution is small relative to the transport sweep [14], and

3. the local zone, direction, group matrix inversion required for DFEM radiation transport spatial discretizations is the sweep computational bottleneck [5].

A transport sweep in 1-D requires

$$
\frac{N_{u n k}}{P+1}
$$

local matrix inversions of a $(P+1) \times(P+1)$ dense matrix, and we use Gaussian elimination to solve the local DFEM system of equations, requiring FLOPs proportional to the cube of the matrix dimension [31], $(P+1)^{3}$. In a computing 
environment dominated by a FLOP bottleneck, the time to solution, $t_{\text {sol }}$, is proportional to:

$$
t_{\text {sol }} \propto \frac{N_{u n k}}{P+1}(P+1)^{3} \propto(P+1)^{2} .
$$

In two and three spatial dimensions, assuming $Q_{P}$ DFEM, $t_{s o l}$ would be proportional to $(P+1)^{4}$ and $(P+1)^{6}$, respectively. Though computationally optimized works do not see slow down proportional to $(P+1)^{6}$ they still see slow down relative to lower order methods, and the slowdown increases as DFEM order is increased $[5,30]$.

\section{Conclusions}

We have applied arbitrary stage count SDIRK time integration and arbitrary trial space degree DFEM to the grey, discrete ordinates thermal radiative transfer equations. Additionally, we have applied the modified interior penalty diffusion discretization to accelerate the iterative convergence of the grey TRT equations. Using the method of manufactured solutions, we have shown that applying higher order SDIRK time integration schemes to the grey TRT yields higher order temporal convergence for both the angle integrated intensity and temperature as compared to implicit Euler time integration.

Due to their demonstrated robustness and accuracy applied to linear neutron transport problems [10, 18], we limited our consideration of DFEM schemes to a family of quadrature based self-lumping DFEMs that use either GaussLegendre or Lobatto-Gauss-Legendre quadrature as the DFEM interpolation points. We have considered four total DFEM schemes, SL Gauss, SLXS Gauss, SL Lobatto, and SLXS Lobatto. The SL Gauss and SLXS Gauss schemes used Gauss-Legendre quadrature as the DFEM interpolation points whereas the SL Lobatto and SLXS Lobatto schemes used Lobatto-Gauss-Legendre quadrature as the DFEM interpolation points. The SL Gauss and SL Lobatto schemes assumed zone-wise constant material properties equal to the volumetric average of the spatially varying quantities. SLXS Gauss and SLXS Lobatto explicitly account for the within zone spatial variation of material properties by extending 
the self-lumping quadrature integration of the DFEM moment equation quantities to include the spatial variation of material properties, not just the spatial variation of DFEM basis and trial space polynomials.

Though there is no difference between the SL and SLXS variants of a scheme for problems with zone-wise constant material properties [10], for problems with spatially varying material properties, which includes nearly all thermal radiative transfer problems of science and engineering interest, the assumption of zone-wise constant material properties was shown to result in very undesirable behavior. First, regardless of DFEM trial space degree, for problems with spatially varying material properties, the SL Lobatto and SL Gauss schemes converged the $L_{2}$ error of the temperature solution at most first order in space and converged the $L_{2}$ error of the angle integrated intensity at most second order in space. In contrast, the SLXS Lobatto scheme converged the $L_{2}$ errors in temperature and angle integrated intensity proportional to $\Delta x^{P}$ and $\Delta x^{P+1}$, respectively, where $P$ is the DFEM trial space degree. The SLXS Gauss scheme converged both the errors in temperature and angle integrated intensity proportional to $\Delta x^{P+1}$. Additionally, the assumption of zone-wise constant opacities for a Marshak wave problem with spatially varying opacity (due to the temperature dependence of opacity), was shown to cause the SL Lobatto and SL Gauss schemes to yield temperature solutions with large, non-monotonic discontinuities regardless of DFEM trial space degree, spatial resolution, or DFEM interpolation point type. The SLXS Lobatto and SLXS Gauss schemes did not exhibit these discontinuities in the temperature profile.

An obvious next step for this work is to apply the SLXS Lobatto and SLXS Gauss schemes to higher spatial dimensions and multi-frequency discrete ordinates thermal radiative transfer simulations. Additionally, we intend to examine adaptive time step selecting techniques for use with our higher order DFEM and multiple stage count SDIRK time integration techniques, comparing the performance and accuracy of traditional temperature change criteria of [8] versus embedded SDIRK methods [32] that can automatically estimate temporal error. Finally, we intend to examine the effects on accuracy and robustness of 
not assuming the Planck function spatial dependence is within the DFEM trial space.

\section{Acknowledgments}

The work of P. G. Maginot was performed under the auspices of the U.S. Department of Energy by Lawrence Livermore National Laboratory under Contract DE-AC52-07NA27344. The work of J. C. Ragusa and J. E. Morel was supported in part by the Center for Exascale Radiation Transport, under Department of Energy, National Nuclear Security Administration, Award Number DE-NA0002376.

\section{References}

[1] Y. Wang, J. C. Ragusa, A high-order discontinuous Galerkin method for the $S_{N}$ transport equations on 2D unstructured triangular meshes, Annals of Nuclear Energy 36 (7) (2009) 931-939.

[2] S. H. Fuller, L. I. Millett (Eds.), The Future of Computing Performance: Game Over or Next Level, National Academies Press, 2011.

[3] S. H. Langer, I. Karlin, V. A. Dobrev, M. L. Stowell, M. E. Kumbera, Performance analysis and optimization for BLAST, a high order finite element hydro code, Tech. Rep. LLNL-PROC-666382, Lawrence Livermore National Laboratory (2015).

[4] T. Kolev, R. Anderson, T. Brunner, J. Cerveny, V. Dobrev, A. Grayver, I. Karlin, R. Rieben, Scalable high order finite elements for compressible hydrodynamics, Tech. Rep. LLNL-PRES-680308, Lawrence Livermore National Laboratory (2015).

[5] P. G. Maginot, P. N. Brown, A. J. Kunen, T. S. Bailey, The improved simple corner balance method and efforts to enhance its computational performance, Transactions of the American Nuclear Society. 
[6] A. G. Petschek, R. E. Williamson, J. K. W. Jr., The pentetration of radiation with constant driving temperature, Tech. Rep. LAMS-2421, Los Alamos Scientific Lab (1960).

[7] C. C. Ober, J. N. Shadid, Studies on the accuracy of time-integration methods for the radiation-diffusion equations, Journal of Computational Physics 195 (2004) 743-772.

[8] J. D. Edwards, J. E. Morel, D. A. Knoll, Nonlinear variants of the TR/BDF2 method for thermal radiative diffusion, Journal of Computational Physics 230 (2011) 1198-1214.

[9] J. E. Morel, B. T. Adams, T. Noh, J. M. McGhee, T. M. Evans, T. J. Urbatsch, Spatial discretizations for self-adjoint forms of the radiative transfer equations, Journal of Computational Physics 214 (2006) 12-40.

[10] P. G. Maginot, J. C. Ragusa, J. E. Morel, Accurate methods for DFEM $S_{N}$ transport in slab geometry for non-piecewise constant cross section problems, Annals of Nuclear Energy 73 (2014) 506-526.

[11] J. E. Morel, T. A. Wareing, K. Smith, A linear-discontinuous spatial differencing scheme for $S_{N}$ radiative transfer calculations, Journal of Computational Physics 128 (1996) 445-462.

[12] R. Alexander, Diagonally implicit Runge-Kutta methods for stiff O.D.E.'s, SIAM Journal of Numerical Analysis 14 (6) (1977) 1006-1021.

[13] Y. Wang, J. C. Ragusa, Diffusion synthetic acceleration for high-order discontinuous finite element $S_{N}$ transport schemes and application to locally refined unstructured meshes, Nuclear Science and Engineering 166 (2010) $145-166$.

[14] B. Turcksin, J. C. Ragusa, A diffusion synthetic acceleration scheme for rectangular geometries based on bilinear discontinuous finite elements, in: International Conference on Mathematics and Computational methods, Applied to Nuclear Science and Engineering, Sun Valley, Idaho, 2013. 
[15] S. P. Nørsett, P. G. Thomsen, Local error control in SDIRK-methods, BIT 26 (1986) 100-113.

[16] P. A. Raviart, The use of numerical integration in finite element methods for solving parabolic equations, in: Conference on Numerical Analysis, RIANA 1972, 1972, pp. 233-264.

[17] V. Thomee, Galerkin Finite Element Methods for Parabolic Problems, Springer, New York, 1997.

[18] P. G. Maginot, J. C. Ragusa, J. E. Morel, Lumping techniques for DFEM transport in $S_{N}$ transport in slab geometry, Nuclear Science and Engineering 179 (2) (2015) 148-163.

[19] E. E. Lewis, W. F. Miller, Computational Methods of Neutron Transport, American Nuclear Society, La Grange Park, IL, 1993.

[20] E. M. Gelbard, L. A. Hageman, The synthetic method as applied to $S_{N}$ equations, Nuclear Science and Engineering 37 (2) (1969) 288-298.

[21] E. W. Larsen, Unconditionally stable diffusion-synthetic acceleration methods for the slab geometry discrete ordinates equations, Nuclear Science and Engineering 82 (1982) 47-63.

[22] T. A. Wareing, E. W. Larsen, M. L. Adams, Diffusion accelerated discontinuous finite element schemes for the $S_{N}$ equations in slab and X-Y geometries, in: Advances in Mathematics, Computations, and Reactor Physics, Pittsburgh, Pennsylvania, 1991.

[23] M. L. Adams, W. R. Martin, Diffusion synthetic acceleration of discontinuous finite element transport iterations, Nuclear Science and Engineering 111 (1992) 145-167.

[24] J. S. Warsa, T. A. Wareing, J. E. Morel, Fully consistent diffusion synthetics acceleration of linear discontinuous $S_{N}$ transport discretization on unstructured tetrahedral meshes, Nuclear Science and Engineering 141 (2002) 236-251. 
[25] J. Nocedal, Numerical Optimization, 2nd Edition, Springer, New York, NY, 2006.

[26] M. L. Adams, E. W. Larsen, Fast iterative methods for discrete-ordinates particle transport calculations, Progress in Nuclear Energy 40 (1) (2002) $3-159$.

[27] B. Su, G. L. Olson, An analytical benchmark for non-equilibrium radiative transfer in an isotropically scattering medium, Annals of Nuclear Engergy 24 (13) (1997) 1035-1055.

[28] C. Lingus, Analytical tests cases for neutrons and radiation transport codes, in: Second Conference on Transport Theory, United States Atomic Energy Commision- Division of Technical Information, Los Alamos, New Mexico, 1971, pp. 655-659.

[29] K. Salari, P. Knupp, Code verification by the method of manufactured solutions, Tech. Rep. SAND2000-1444, Sandia National Labs (2000).

[30] V. A. Dobrev, I. Karlin, T. Kolev, R. Rieben, Scalable high-order multimaterial ALE simulations, Poster LLNL-POST-676385, Lawrence Livermore National Laboratory (2015).

[31] J. D. Hoffman, Numerical Methods for Engineers and Scientists, 2nd Edition, Marcel Dekker, 2001.

[32] S. P. Nørsett, P. G. Thomsen, Embedded SDIRK methods of basic order three, BIT 24 (1984) 634-646.

\section{Appendix A. Butcher Tableaux}

The Butcher tableaux for the implicit Euler scheme can be written as:

\begin{tabular}{c|c|c} 
Stage & $\gamma_{i}$ & $\alpha_{i j}$ \\
\hline 1 & 1 & 1 \\
\hline$\beta_{i}$ & & 1
\end{tabular}.


The SDIRK 2-2 scheme of Alexander [12] has the following Butcher tableaux,

\begin{tabular}{c|c|cc} 
Stage & $\gamma_{i}$ & $\alpha_{i j}$ & \\
\hline 1 & $1-\frac{\sqrt{2}}{2}$ & $1-\frac{\sqrt{2}}{2}$ & 0 \\
2 & 1 & $\frac{\sqrt{2}}{2}$ & $1-\frac{\sqrt{2}}{2}$ \\
\hline$\beta_{i}$ & & $\frac{\sqrt{2}}{2}$ & $1-\frac{\sqrt{2}}{2}$
\end{tabular}

and the SDIRK 3-3 [12] Butcher tableaux is:

\begin{tabular}{c|c|ccc} 
Stage & $\gamma_{i}$ & \multicolumn{3}{|c}{$\alpha_{i j}$} \\
\hline 1 & $\kappa$ & $\kappa$ & & \\
2 & $\frac{1+\kappa}{2}$ & $\frac{1-\kappa}{2}$ & $\kappa$ & \\
3 & 1 & $\delta$ & $\zeta$ & $\kappa$ \\
\hline$\beta_{i}$ & & $\delta$ & $\zeta$ & $\kappa$
\end{tabular}

with

$$
\begin{aligned}
\kappa & =0.435866521508459 \\
\delta & =\frac{-6 \gamma^{2}+16 \gamma-1}{4} \\
\zeta & =\frac{6 \gamma^{2}-20 \gamma+5}{4}
\end{aligned}
$$

\section{Appendix B. Linearization of the TRT Equations in Temperature}

\section{Appendix B.1. Complete Manipulation}

We now give a more complete derivation of the linearized, grey thermal radiative transfer equations highlighted in Section 2. We begin by repeating Eqs. (35) here,

$$
\begin{aligned}
\vec{I}^{i}=\vec{I}^{n}+\Delta t \sum_{j=1}^{i-1} & \alpha_{i j} \vec{k}_{I}^{j}+\frac{\alpha_{i i} \Delta t c}{4 \pi} \mathbf{M}^{-1} \mathbf{R}_{\sigma_{s}} \vec{\phi}^{i} \\
& +\alpha_{i i} \Delta t c \mathbf{M}^{-1} \mathbf{R}_{\sigma_{a}}\left[\vec{B}^{*}+\mathbf{D}^{*}\left(\vec{T}^{i}-\vec{T}^{*}\right)\right] \\
& \quad+\alpha_{i i} \Delta t c \mathbf{M}^{-1}\left[\mu_{d} I_{i n} \vec{u}-\mathbf{R}_{\sigma_{t}} \vec{I}^{i}-\mu_{d} \mathbf{G} \vec{I}+\vec{Q}_{I}^{i}\right]
\end{aligned}
$$




$$
\begin{aligned}
\vec{T}^{i}=\vec{T}^{n} & +\Delta t \sum_{j=1}^{i-1} \alpha_{i j} \vec{k}_{T}^{j} \\
& \quad+\alpha_{i i} \Delta t \mathbf{R}_{C_{v}}^{-1}\left(\mathbf{R}_{\sigma_{a}}\left\{\vec{\phi}^{i}-4 \pi\left[\vec{B}^{*}+\mathbf{D}^{*}\left(\vec{T}^{i}-\vec{T}^{*}\right)\right]\right\}+\vec{Q}_{T}^{i}\right) .
\end{aligned}
$$

First, we move all $\vec{T}^{i}$ terms of Eq. (B.1b) to the left hand side,

$$
\begin{aligned}
\vec{T}^{i}+4 \pi \alpha_{i i} \Delta t \mathbf{R}_{C_{v}}^{-1} \mathbf{R}_{\sigma_{a}} \mathbf{D}^{*} \vec{T}^{i} & =\vec{T}^{n}+\Delta t \sum_{j=1}^{i-1} \alpha_{i j} \vec{k}_{T}^{j} \\
& +\alpha_{i i} \Delta t \mathbf{R}_{C_{v}}^{-1}\left\{\mathbf{R}_{\sigma_{a}}\left[\vec{\phi}^{i}-4 \pi\left(\vec{B}^{*}-\mathbf{D}^{*} \vec{T}^{*}\right)\right]+\vec{Q}_{T}^{i}\right\} .
\end{aligned}
$$

Grouping the $\vec{T}^{i}$ on the left hand side and extracting the $\vec{T}^{*}$ terms from the right hand side yields

$$
\begin{aligned}
& {\left[\mathbf{I}+4 \pi \alpha_{i i} \Delta t \mathbf{R}_{C_{v}}^{-1} \mathbf{R}_{\sigma_{a}} \mathbf{D}^{*}\right] \vec{T}^{i}=\vec{T}^{n}+\Delta t \sum_{j=1}^{i-1} \alpha_{i j} \vec{k}_{T}^{j} } \\
&+\alpha_{i i} \Delta t \mathbf{R}_{C_{v}}^{-1}\left\{\mathbf{R}_{\sigma_{a}}\left[\vec{\phi}^{i}-4 \pi \vec{B}^{*}\right]+\vec{Q}_{T}^{i}\right\} \\
& \quad+4 \pi \alpha_{i i} \Delta t \mathbf{R}_{C_{v}}^{-1} \mathbf{R}_{\sigma_{a}} \mathbf{D}^{*} \vec{T}^{*} .
\end{aligned}
$$

Adding a zero vector,

$$
\mathbf{I}\left[\vec{T}^{*}-\vec{T}^{*}\right]
$$

to the right hand side, then multiplying by $\left[\mathbf{I}+4 \pi \alpha_{i i} \Delta t \mathbf{R}_{C_{v}}^{-1} \mathbf{R}_{\sigma_{a}} \mathbf{D}^{*}\right]^{-1}$ gives

$$
\begin{aligned}
& \vec{T}^{i}=\vec{T}^{*}+\left[\mathbf{I}+4 \pi \alpha_{i i} \Delta t \mathbf{R}_{C_{v}}^{-1} \mathbf{R}_{\sigma_{a}} \mathbf{D}^{*}\right]^{-1}\left[\vec{T}^{n}-\vec{T}^{*}+\Delta t \sum_{j=1}^{i-1} \alpha_{i j} \vec{k}_{T}^{j}\right] \\
& \quad+\alpha_{i i} \Delta t\left[\mathbf{I}+4 \pi \alpha_{i i} \Delta t \mathbf{R}_{C_{v}}^{-1} \mathbf{R}_{\sigma_{a}} \mathbf{D}^{*}\right]^{-1} \mathbf{R}_{C_{v}}^{-1}\left\{\mathbf{R}_{\sigma_{a}}\left[\vec{\phi}^{i}-4 \pi \vec{B}^{*}\right]+\vec{Q}_{T}^{i}\right\}
\end{aligned}
$$

Introducing the shortcut notation of Eq. (37),

$$
\mathbf{C}^{i}=\left[\mathbf{I}+4 \pi \Delta t \alpha_{i i} \mathbf{R}_{C_{v}}^{-1} \mathbf{R}_{\sigma_{a}} \mathbf{D}^{*}\right]^{-1},
$$

gives the stage $i$ temperature update equation, Eq. (36),

$$
\begin{aligned}
& \vec{T}^{i}=\vec{T}^{*}+\mathbf{C}^{i}\left[\vec{T}^{n}-\vec{T}^{*}+\Delta t \sum_{j=1}^{i-1} \alpha_{i j} \vec{k}_{T}^{j}\right] \\
& +\Delta t \alpha_{i i} \mathbf{C}^{i} \mathbf{R}_{C_{v}}^{-1}\left[\mathbf{R}_{\sigma_{a}}\left(\vec{\phi}^{i}-4 \pi \vec{B}^{*}\right)+\vec{Q}_{T}^{i}\right] .
\end{aligned}
$$


Derivation of the pseudo-fission form linear transport equation of Eq.

is more straightforward than the manipulation required to achieve Eq. (B.7). We begin by inserting Eq. (B.7) into Eq. (B.1a)

$$
\begin{aligned}
\vec{I}^{i}=\vec{I}^{n} & +\Delta t \sum_{j=1}^{i-1} \alpha_{i j} \vec{k}_{I}^{j} \\
& +\alpha_{i i} \Delta t c \mathbf{M}^{-1}\left[\frac{1}{4 \pi} \mathbf{R}_{\sigma_{s}} \vec{\phi}^{i}-\mathbf{R}_{\sigma_{t}} \vec{I}^{i}+\mu_{d} I_{i n} \vec{u}-\mu_{d} \mathbf{G} \vec{I}^{i}+\vec{Q}_{I}^{i}\right] \\
& +\alpha_{i i} \Delta t c \mathbf{M}^{-1} \mathbf{R}_{\sigma_{a}}\left\{\vec{B}^{*}+\mathbf{D}^{*} \mathbf{C}^{i}\left[\vec{T}^{n}-\vec{T}^{*}+\Delta t \sum_{j=1}^{i-1} \alpha_{i j} \vec{k}_{T}^{j}\right]\right\} \\
& +\alpha_{i i} \Delta t c \mathbf{M}^{-1} \mathbf{R}_{\sigma_{a}} \mathbf{D}^{*} \Delta t \alpha_{i i} \mathbf{C}^{i} \mathbf{R}_{C_{v}}^{-1}\left[\mathbf{R}_{\sigma_{a}}\left(\vec{\phi}^{i}-4 \pi \vec{B}^{*}\right)+\vec{Q}_{T}^{i}\right] .
\end{aligned}
$$

Multiplying by $\frac{1}{\alpha_{i i} c \Delta t} \mathbf{M}$ and moving all $\vec{I}^{i}$ terms to the left hand side yields:

$$
\begin{array}{r}
\mu_{d} \mathbf{G} \vec{I}^{i}+\left(\mathbf{R}_{\sigma_{t}}+\frac{1}{\alpha_{i i} c \Delta t} \mathbf{M}\right) \vec{I}^{i}=\frac{1}{\alpha_{i i} c \Delta t}\left[\mathbf{M} \vec{I}^{n}+\Delta t \mathbf{M} \sum_{j=1}^{i-1} \alpha_{i j} \vec{k}_{I}^{j}\right] \\
+\frac{1}{4 \pi} \mathbf{R}_{\sigma_{s}} \vec{\phi}^{i}+\mu_{d} I_{i n} \vec{u}+\vec{Q}_{I}^{i}+\mathbf{R}_{\sigma_{a}}\left\{\vec{B}^{*}+\mathbf{D}^{*} \mathbf{C}^{i}\left[\vec{T}^{n}-\vec{T}^{*}+\Delta t \sum_{j=1}^{i-1} \alpha_{i j} \vec{k}_{T}^{j}\right]\right\} \\
+\alpha_{i i} \Delta t \mathbf{R}_{\sigma_{a}} \mathbf{D}^{*} \mathbf{C}^{i} \mathbf{R}_{C_{v}}^{-1}\left[\mathbf{R}_{\sigma_{a}}\left(\vec{\phi}^{i}-4 \pi \vec{B}^{*}\right)+\vec{Q}_{T}^{i}\right] .
\end{array}
$$

We then isolate all $\vec{\phi}^{i}$ terms:

$$
\begin{aligned}
\mu_{d} \mathbf{G} \vec{I}^{i}+\left(\mathbf{R}_{\sigma_{t}}+\right. & \left.\frac{1}{\alpha_{i i} c \Delta t} \mathbf{M}\right) \vec{I}^{i}=\frac{1}{\alpha_{i i} c \Delta t}\left[\mathbf{M} \vec{I}^{n}+\Delta t \mathbf{M} \sum_{j=1}^{i-1} \alpha_{i j} \vec{k}_{I}^{j}\right] \\
+\frac{1}{4 \pi} \mathbf{R}_{\sigma_{s}} \vec{\phi}^{i}+\alpha_{i i} \Delta t \mathbf{R}_{\sigma_{a}} \mathbf{C}^{i} \mathbf{R}_{C_{v}}^{-1} \mathbf{R}_{\sigma_{a}} \vec{\phi}^{i} & \\
+\mu_{d} I_{i n} \vec{u}+\vec{Q}_{I}^{i}+\mathbf{R}_{\sigma_{a}} & \left\{\vec{B}^{*}+\mathbf{D}^{*} \mathbf{C}^{i}\left[\vec{T}^{n}-\vec{T}^{*}+\Delta t \sum_{j=1}^{i-1} \alpha_{i j} \vec{k}_{T}^{j}\right]\right\} \\
& -\alpha_{i i} \Delta t \mathbf{R}_{\sigma_{a}} \mathbf{D}^{*} \mathbf{C}^{i} \mathbf{R}_{C_{v}}^{-1}\left[\mathbf{R}_{\sigma_{a}} 4 \pi \vec{B}^{*}-\vec{Q}_{T}^{i}\right] .
\end{aligned}
$$

Using the definitions of Eq. (39) [repeated below],

$$
\begin{gathered}
\overline{\bar{\nu}}^{i}=4 \pi \Delta t a_{i i} \mathbf{R}_{\sigma_{a}} \mathbf{D}^{*} \mathbf{C}^{i} \mathbf{R}_{C_{v}}^{-1} \\
\mathbf{R}_{\sigma_{\tau}}^{i}=\mathbf{R}_{\sigma_{t}}+\frac{1}{c \Delta t \alpha_{i i}} \mathbf{M}
\end{gathered}
$$




$$
\begin{aligned}
\vec{\xi}^{\imath}=\frac{1}{c \Delta t \alpha_{i i}} \mathbf{M} \vec{I}^{n} & +\frac{1}{c \alpha_{i i}} \mathbf{M} \sum_{j=1}^{i-1} \alpha_{i j} \vec{k}_{I}^{j}+\mathbf{R}_{\sigma_{a}} \vec{B}^{*}+\vec{Q}_{I}^{i} \\
& +\mathbf{R}_{\sigma_{a}} \mathbf{D}^{*} \mathbf{C}^{i}\left[\vec{T}^{n}-\vec{T}^{*}+\Delta t \sum_{j=1}^{i-1} \alpha_{i j} \vec{k}_{T}^{j}\right] \\
& +\Delta t \alpha_{i i} \mathbf{R}_{\sigma_{a}} \mathbf{D}^{*} \mathbf{C}^{i} \mathbf{R}_{C_{v}}^{-1}\left[\vec{Q}_{T}^{i}-4 \pi \mathbf{R}_{\sigma_{a}} \vec{B}^{*}\right]
\end{aligned}
$$

Eq. (B.10) becomes

$$
\mu_{d} \mathbf{G} \vec{I}^{i}+\mathbf{R}_{\sigma_{\tau}}^{i} \vec{I}^{\imath}=\frac{1}{4 \pi} \mathbf{R}_{\sigma_{s}} \vec{\phi}^{i}+\frac{1}{4 \pi} \overline{\bar{\nu}}_{i} \mathbf{R}_{\sigma_{a}} \vec{\phi}^{i}+\vec{\xi}^{\imath}+\mu_{d} I_{i n} \vec{u} .
$$

Equation (B.12) is the spatially discretized, pseudo-fission, linear, radiation transport equation for a given temperature iterate, $T^{*}$.

Appendix B.2. Effective Scattering Matrix

We now define the scattering matrix ratio matrix, $\mathbf{S}^{i}$ for implicit Euler time differencing and SL Lobatto spatial discretization. With the single stage implicit Euler scheme, $\mathbf{S}^{i}=\mathbf{S}^{1}$. Seeking to evaluate the definition of Eq. (58),

$$
\mathbf{S}^{i}=\left[\mathbf{R}_{\sigma_{\tau}}^{1}\right]^{-1}\left[\overline{\bar{\nu}}^{1} \mathbf{R}_{\sigma_{a}}+\mathbf{R}_{\sigma_{s}}\right],
$$

we first define $\mathbf{R}_{\sigma_{a}}, \mathbf{R}_{\sigma_{s}}$, and $\mathbf{R}_{\sigma_{\tau}}^{1}$ :

$$
\begin{gathered}
\mathbf{R}_{\sigma_{a}}=\frac{\Delta x_{z} \hat{\sigma}_{a}}{2}\left[\begin{array}{ll}
1 & 0 \\
0 & 1
\end{array}\right] \\
\mathbf{R}_{\sigma_{s}}=\frac{\Delta x_{z} \hat{\sigma}_{s}}{2}\left[\begin{array}{ll}
1 & 0 \\
0 & 1
\end{array}\right] \\
\mathbf{R}_{\sigma_{\tau}}^{1}=\left(\hat{\sigma}_{a}+\hat{\sigma}_{s}+\frac{1}{c \Delta t}\right) \frac{\Delta x_{z}}{2}\left[\begin{array}{ll}
1 & 0 \\
0 & 1
\end{array}\right] .
\end{gathered}
$$

The definition of $\overline{\bar{\nu}}^{i}$,

$$
4 \pi \Delta t \alpha_{i i} \mathbf{R}_{\sigma_{a}} \mathbf{D}^{*} \mathbf{C}^{i} \mathbf{R}_{C_{v}}^{-1},
$$

additionally requires the definition of $\mathbf{D}^{*}, \mathbf{R}_{C_{v}}^{-1}$,

$$
\mathbf{D}^{*}=\left[\begin{array}{cc}
\left.\frac{\partial B}{\partial T}\right|_{T=T^{*} z, 1} & 0 \\
0 & \left.\frac{\partial B}{\partial T}\right|_{T=T^{*} z, 2}
\end{array}\right]
$$




$$
\mathbf{R}_{C_{v}}^{-1}=\frac{2}{\Delta x_{z} \bar{C}_{v}}\left[\begin{array}{ll}
1 & 0 \\
0 & 1
\end{array}\right]
$$

and $\mathbf{C}^{1}$,

$$
\begin{gathered}
\mathbf{C}^{1}=\left[\mathbf{I}+4 \pi \Delta t \mathbf{R}_{C_{v}}^{-1} \mathbf{R}_{\sigma_{a}} \mathbf{D}^{*}\right]^{-1} \\
\mathbf{C}^{1}=\left[\begin{array}{cc}
1+\left.4 \pi \Delta t \frac{\hat{\sigma}_{a}}{\bar{C}_{v}} \frac{\partial B}{\partial T}\right|_{T=T^{*} z, 1} & 0 \\
0 & 1+\left.4 \pi \Delta t \frac{\hat{\sigma}_{a}}{\bar{C}_{v}} \frac{\partial B}{\partial T}\right|_{T=T^{*} z, 1}
\end{array}\right] \\
\mathbf{C}^{1}=\left[\begin{array}{cc}
\frac{\bar{C}_{v}}{\bar{C}_{v}+\left.4 \pi \Delta t \sigma_{a} \frac{\partial B}{\partial T}\right|_{T=T_{z, 1}^{*}}} & 0 \\
0 & \frac{\bar{C}_{v}}{\bar{C}_{v}+\left.4 \pi \Delta t \sigma_{a} \frac{\partial B}{\partial T}\right|_{T=T_{z, 2}^{*}}}
\end{array}\right]
\end{gathered}
$$

Using Eqs. (B.14) and Eqs. (B.17), we evaluate $\overline{\bar{\nu}}^{1}$,

$$
\overline{\bar{\nu}}^{1}=\left[\begin{array}{cc}
\left.4 \pi \Delta t \bar{\sigma}_{a} \frac{\partial B}{\partial T}\right|_{T=T_{z, 1}^{*}} & 0 \\
\overline{\bar{C}_{v}+\left.4 \pi \Delta t \sigma_{a} \frac{\partial B}{\partial T}\right|_{T=T_{z, 1}^{*}}} & \left.4 \pi \Delta t \bar{\sigma}_{a} \frac{\partial B}{\partial T}\right|_{T=T_{z, 2}^{*}} \\
0 & \frac{\bar{C}_{v}+\left.4 \pi \Delta t \sigma_{a} \frac{\partial B}{\partial T}\right|_{T=T_{z, 2}^{*}}}{0}
\end{array}\right],
$$

then evaluate $\mathbf{S}^{1}$,

$$
\begin{aligned}
& \mathbf{S}^{1}=\left(\frac{c \Delta t}{1+c \Delta t \bar{\sigma}_{t}}\right) \operatorname{diag}\left[\bar{\sigma}_{s}+\bar{\sigma}_{a}\left(\frac{\left.4 \pi \Delta t \bar{\sigma}_{a} \frac{\partial B}{\partial T}\right|_{T=T_{z, 1}^{*}}}{\bar{C}_{v}+\left.4 \pi \Delta t \bar{\sigma}_{a} \frac{\partial B}{\partial T}\right|_{T=T_{z, 1}^{*}}}\right),\right. \\
& \left.\bar{\sigma}_{s}+\bar{\sigma}_{a}\left(\frac{\left.4 \pi \Delta t \bar{\sigma}_{a} \frac{\partial B}{\partial T}\right|_{T=T_{z, 2}^{*}}}{\bar{C}_{v}+\left.4 \pi \Delta t \bar{\sigma}_{a} \frac{\partial B}{\partial T}\right|_{T=T_{z, 2}^{*}}}\right)\right] .
\end{aligned}
$$

